\title{
Generalized Receiver with Parallel Interference Cancellation for Multiuser Detection
}

\author{
VYACHESLAV TUZLUKOV \\ Department of Technical Maintenance of Aviation and Radio Electronic Equipment \\ Belarusian State Aviation Academy \\ 77, Uborevicha Str., 220096 Minsk \\ BELARUS
}

\begin{abstract}
Parallel interference cancellation is considered as a simple yet effective multiuser detector for direct -sequence code-division multiple-access (DS-CDMA) systems. However, system performance be deteriorated due to unreliable interference cancellation in the early stages. Thus, a detector with the partial parallel interference cancellation in which the partial cancellation factors are introduced to control the interference cancellation level has been developed as a remedy. Although the partial cancellation factors are crucial, complete solutions for their optimal values are not available. In this paper, we consider a two-stage decoupled generalized receiver with the partial parallel interference cancellation. Using the minimum bit error rate $(B E R)$ criterion, we derive a complete set of optimal partial cancellation factors. This includes the optimal partial cancellation factors for periodic and aperiodic spreading codes in channels with the additive white Gaussian noise and multipath channels. Simulation results demonstrate that the considered theoretical optimal partial cancellation factors agree closely with empirical ones. The proposed two-stage generalized receiver with the partial parallel interference cancellation using the derived optimal partial cancellation factors outperforms not only a two-stage, but also a three-stage conventional generalized receiver with the full parallel interference cancellation.
\end{abstract}

Key-Words: - Generalized receiver, direct-sequence code-division multiple-access (DS-CDMA), multiple access interference (MAI), multiuser detection, parallel interference cancellation.

Received: May 9, 2021. Revised: November 3, 2021. Accepted: November 15, 2021. Published: December 9, 2021.

\section{Introduction}

Direct-sequence code-division multiple-access (DSCDMA) is considered as promising technique in cellular and personal communications. Conventional matched-filter receivers suffer from multiple access interference (MAI) and the near-far effect. A maximum-likelihood multiuser detector was proposed in [1] to mitigate these problems. Unfortunately, the computational complexity of this approach grows exponentially with the user number, prohibiting its practical applications.

Suboptimum receivers were then considered in [2]-[7] to reduce the computational complexity. The decorrelator, being a linear receiver, can effectively eliminate the MAI. However, it may greatly enhance the noise [8]. The linear minimum mean square error (LMMSE) detector discussed in [8]-[11] is an improvement to the decorrelator and represents a compromise between interference suppression and noise enhancement. Although these suboptimal approaches are much simpler than the optimal solution, they require matrix inversion operations that are undesirable in practice.

In addition to the aforementioned linear detectors, another category of interest is the subtractive-type interference cancellation method. Cancellation of this type involves only vector operations making it a good candidate for real-world implementation. For particular desired user, the subtractive-type canceller estimates the interference from other users, regenerates it, and cancels it from the received signal. This cancellation process can be carried out for each interfering user either successively, i.e., successive interference cancellation [12]-[14] or in parallel, i.e. parallel interference cancellation [15]-[19]. Implementation of the successive interference cancellation allows us to estimate and cancel MAI one by one while under employment of parallel interference cancellation all interferences are cancelled simultaneously.

Using the successive interference cancellation it is possible to reach better performance in comparison with the parallel interference cancellation. However, its computational complexity is higher and processing delays are larger. There are two types of parallel interference cancellation classified according to the tentative decision devices used in each stage, namely, the hard-decision parallel interference cancellation [15]-[22] and the soft-decision parallel interference cancellation [16], [20], [23][25]. It has been observed that the hard-decision parallel interference cancellation can provide better 
performance in comparison with the soft-decision parallel interference cancellation [26].

Conventional receivers with the parallel interference cancellation permit us a full MAI cancellation. One problem associated with this approach is that the MAI estimate may not be reliable in the earlier cancelling stages. This makes the parallel interference cancellation less effective when the number of users is large. The partial parallel interference cancellation detector has been proposed in which partial cancellation factors are introduced to control the interference cancellation level as a remedy [20], [21], [27]. However, theoretical analysis of the partial hard-decision parallel interference cancellation is difficult due to the nonlinear decision devices used in the receiver. Consequently, the optimal partial cancellation factors are usually obtained either by training via the least mean square adaptive algorithm [28] and [29] or theoretical derivation under some simplifying assumptions [30].

It is well known that the least mean square algorithm is simple but converges slowly. The approaches in [28]-[35] may not be adequate in fast fading environments. On the other hand, the optimal partial cancellation factors derived in [26], [37] are only valid when the number of users is small. The optimal partial cancellation factors for soft-decision parallel interference cancellation are derived in [37]. However, these results can be applied only to a perfect power control scenario. As mentioned above, the hard-decision parallel interference cancellation allows us to get better performance in comparison with the partial soft-decision parallel interference cancellation. Although this seems intuitively reasonable, it may not be true. The previous investigation demonstrate that for a two-stage canceller incorporated into the generalized receiver [34] with the optimal partial soft-decision parallel interference cancellation can deliver the similar performance as the generalized receiver based on the optimal partial hard-decision parallel interference cancellation.

A complete partial parallel interference cancellation requires $K(K-1)$ partial cancellation factors, where $K$ is the number of users and the computational complexity is high. Simplified the partial parallel interference cancellation has been proposed, in which only $K$ partial cancellation factors are needed. Two structures are commonly used for simplified partial parallel interference cancellation. We call them the coupled and decoupled structures. In the coupled structure, each user output is related to all $K$ partial cancellation factors [28], [29] while in the decoup-led structure, each user output is only related to a specific partial cancellation factor.
The hard-decision parallel interference cancellation mentioned above uses the coupled structure. While the partial soft-decision parallel interference cancellation described in [21] used a decoupled structure, the optimal partial cancellation factors were not derived. A complete comparison of these two structures is not available in the literature. Our primary study [26] demonstrates that both receivers' structures employing the optimal partial cancellation factors have the similar performance. Since optimizing one partial cancellation factor is much easier than optimizing $K$ partial cancellation factors, it is preferable to deal with the decoupled structure.

In the present paper, we focus on the generalized receiver constructed based on the generalized approach to signal processing in noise [38]-[41] with twostage partial soft-decision parallel interference cancellation using a decoupled structure. Our motivation to use the two-stage signal processing is that it requires a low computational complexity and is particularly suitable for real-world implementation. As indicated in [21] that in higher stage processing, the partial cancellation factors will approach unity for stages greater than two. In other words, the partial cancellation factors in the second stage will dominate system performance.

We first consider the additive white Gaussian noise channel and derive the optimal partial cancellation factors for systems employing periodic codes. The criterion for optimization is the bit error rate $(B E R)$ performance. We then extend the result to systems with aperiodic spreading codes. Finally, we consider the optimal partial cancellation factors with multipath channels. Simulation results show that the performance of the proposed theoretical optimal partial cancellation factors is close to that of empirical ones. In addition, the optimal two-stage partial softdecision parallel interference cancellation outperforms not only the two-stage full soft-decision parallel interference cancellation, but also the three-stage full soft-decision parallel interference cancellation.

The remainder of the present paper is organized as follows. In Section 2 the main functioning principle of the generalized receiver are delivered. In Section 3, we describe the generalized receiver structures with the two-stage full and partial soft-decision parallel interference cancellation. In Sections 4 and 5 , we derive the optimal partial cancellation factors with periodic and aperiodic codes, both in the additive white Gaussian noise and multipath channels. Simulation results are presented and discussed in Section 6. Finally, the conclusions are given in Section 7. 


\section{Generalized Receiver}

The generalized receiver is constructed in accordance with the generalized approach to signal processing in noise [38]-[41]. The generalized approach to signal processing in noise introduces an additional noise source that does not carry any information about the parameters of desired transmitted signal with the purpose to improve the signal processing system performance. This additional noise can be considered as the reference noise without any information about the parameters of the signal to be detected.

The jointly sufficient statistics of the mean and variance of the likelihood function is obtained under the generalized approach to signal processing in noise employment, while the classical and modern signal processing theories can deliver only a sufficient statistics of the mean or variance of the likelihood function. Thus, the generalized approach to signal processing in noise implementation allows us to obtain more information about the parameters of the desired transmitted signal incoming at the generalized receiver input. Owing to this fact, the detectors constructed based on the generalized approach to signal processing in noise technology are able to improve the signal detection performance of signal processing systems in comparison with employment of other conventional detectors.

The generalized receiver (GR) consists of three channels (see Fig.1): the GR correlation detector channel (GR CD) - the preliminary filter (PF), the multipliers 1 and 2, the model signal generator (MSG); the GR energy detector channel (GR ED) the PF, the additional filter (AF), the multipliers 3 and 4 , the summator 1 ; and the GR compensation channel (GR CC) - the summators 2 and 3, the accumulator 1. The threshold apparatus (THRA) device defines the GR threshold.
As we can see from Fig.1, there are two bandpass filters, i.e., the linear systems, at the GR input, namely, the PF and AF. We assume for simplicity that these two filters or linear systems have the same amplitude-frequency characteristics or impulse responses. The AF central frequency is detuned relative to the PF central frequency.

There is a need to note the PF bandwidth is matched with the transmitted signal bandwidth. If the detuning value between the PF and AF central frequencies is more than 4 or 5 times the transmitted signal bandwidth to be detected, i.e. $4 \div 5 \Delta f_{s}$, where $\Delta f_{s}$ is the transmitted signal bandwidth, we can believe that the processes at the PF and AF outputs are uncorrelated because the coefficient of correlation between them is negligible (not more than 0.05). This fact was confirmed experimentally in [42] and [43] independently.

Thus, the transmitted signal plus noise can be appeared at the GR PF output and the noise only is appeared at the GR AF output. The stochastic processes at the GR AF and GR PF outputs present the input stochastic samples from two independent frequency-time regions. If the discrete-time noise $w_{i}[k]$ at the GR PF and GR AF inputs is Gaussian, the discrete-time noise $\zeta_{i}[k]$ at the GR PF output is Gaussian too, and the reference discrete-time noise $\eta_{i}[k]$ at the GR AF output is Gaussian owing to the fact that the GR PF and GR AF are the linear systems and we believe that these linear systems do not change the statistical parameters of the input process. Thus, the GR AF can be considered as a generator of the reference noise with a priori information a "no" transmitted signal (the reference noise sample) [28, Chapter 5]. The noise at the GR PF and GR AF outputs can be presented as

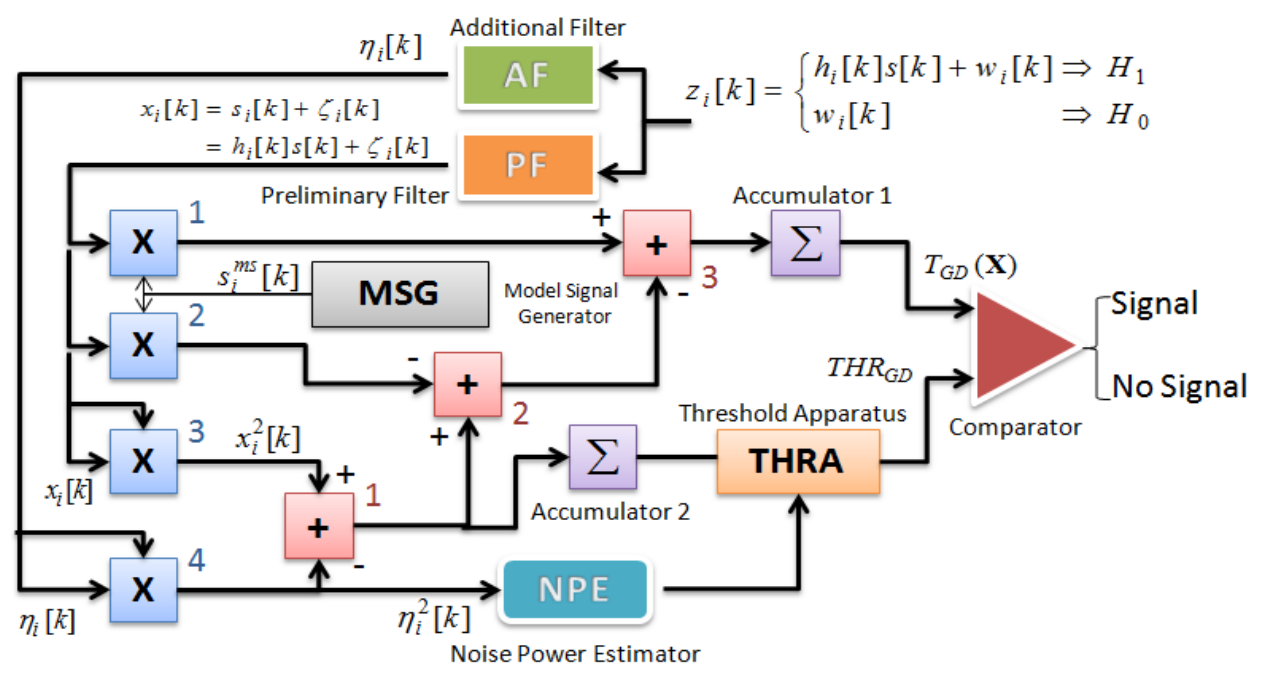

Fig. 1. Generalized receiver. 


$$
\left\{\begin{array}{l}
\zeta_{i}[k]=\sum_{m=-\infty}^{\infty} g_{P F}[m] w_{i}[k-m] ; \\
\eta_{i}[k]=\sum_{m=-\infty}^{\infty} g_{A F}[m] w_{i}[k-m],
\end{array}\right.
$$

where $g_{P F}[m]$ and $g_{A F}[m]$ are the impulse responses of the GR PF and GR AF, respectively.

In a general, under practical implementation of any detector in wireless communication system with sensor array, the bandwidth of the spectrum to be sensed is defined. Thus, the GR AF bandwidth and central frequency can be assigned, too (this bandwidth cannot be used by the transmitted signal because it is out of its spectrum). The case when there are interfering signals within the GR AF bandwidth, the action of this interference on the GR detection performance, and the case of non-ideal condition when the noise at the GR PF and GR AF outputs is not the same by statistical parameters are discussed in [44] and [45].

Under the hypothesis $\mathscr{H}_{1}$ ("a yes" transmitted signal), the GR CD generates the signal component $s_{i}^{m}[k] s_{i}[k]$ caused by interaction between the model signal $s_{i}^{m}[k]$, forming at the MSG output, and the incoming signal $s_{i}[k]$, and the noise component $s_{i}^{m}[k]$ $\times \zeta_{i}[k]$ caused by interaction between the model signal $s_{i}^{m}[k]$ and the noise $\zeta_{i}[k]$ at the PF output. GR ED generates the transmitted signal energy $s_{i}^{2}[k]$ and the random component $s_{i}[k] \zeta_{i}[k]$ caused by interaction between the transmitted signal $s_{i}[k]$ and the noise $\zeta_{i}[k]$ at the PF output. The main purpose of the GR CC is to cancel completely in the statistical sense the GR CD noise component $s_{i}^{m}[k] \zeta_{i}[k]$ and the GR ED random component $s_{i}[k] \zeta_{i}[k]$ based on the same nature of the noise $\zeta_{i}[k]$. The relation between the transmitted signal to be detected $s_{i}[k]$ and the model signal $s_{i}^{m}[k]$ is defined as:

$$
s_{i}^{m}[k]=\mu s_{i}[k],
$$

where $\mu$ is the coefficient of proportionality.

The main functioning condition under the GR employment in any signal processing system including the communication one with radar sensors is the equality between the parameters of the model signal $s_{i}^{m}[k]$ and the incoming signal $s_{i}[k]$, for example, by amplitude. Under this condition it is possible to cancel completely in the statistical sense the noise com- ponent $s_{i}^{m}[k] \zeta_{i}[k]$ of the GR CD and the random component $s_{i}[k] \zeta_{i}[k]$ of the GR ED. Satisfying the GR main functioning condition given by (2), $s_{i}^{m}[k]=$ $s_{i}[k], \mu=1$, we are able to detect the transmitted signal with the high probability of detection at the low $S N R$ and define the transmitted signal parameters with the required high accuracy.

Practical realization of the condition (2) at $\mu \rightarrow 1$ requires increasing in the complexity of GR structure and, consequently, leads us to increasing in computation cost. For example, there is a need to employ the amplitude tracking system or to use the offline data samples processing. Under the hypothesis $\mathscr{H}_{0}$ ("a no" transmitted signal), satisfying the main GR functioning condition (2) at $\mu \rightarrow 1$ we obtain only the background noise $\eta_{i}^{2}[k]-\zeta_{i}^{2}[k]$ at the GR output.

Under practical implementation, the real structure of GR depends on specificity of signal processing systems and their applications, for example, the radar sensor systems, adaptive wireless communication systems, cognitive radio systems, satellite communication systems, mobile communication systems and so on. In the present paper, the GR circuitry (Fig.1) is demonstrated with the purpose to explain the main functioning principles. Because of this, the GR flowchart presented in the paper should be considered under this viewpoint. Satisfying the GR main functioning condition (2) at $\mu \rightarrow 1$, the ideal case, for the wireless communication systems with radar sensor applications we are able to detect the transmitted signal with very high probability of detection and define accurately its parameters.

In the present paper, we discuss the GR implementation in the broadband space-time spreading MC DS-CDMA wireless communication system. Since the presented GR test statistics is defined by the signal energy and noise power, the equality between the parameters of the model signal $s_{i}^{m}[k]$ and transmitted signal to be detected $s_{i}[k]$, in particular by amplitude, is required that leads us to high circuitry complexity in practice.

For example, there is a need to employ the amplitude tracking system or off-line data sample processing. Detailed discussion about the main GR functioning principles if there is no a priori information and there is an uncertainty about the parameters of transmitted signal, i. e., the transmitted signal parameters are random, can be found in [38], [39, Chapter 6, pp.611-621 and Chapter 7, pp. 631-695]. 
The complete matching between the model signal $s_{i}^{m}[k]$ and the incoming signal $s_{i}[k]$, for example by amplitude, is a very hard problem in practice because the incoming signal $s_{i}[k]$ depends on both the fading and the transmitted signal parameters and it is impractical to estimate the fading gain at the low SNR. This matching is possible in the ideal case only. The GD detection performance will be deteriorated under mismatching in parameters between the model signal $s_{i}^{m}[k]$ and the transmitted signal $s_{i}[k]$ and the impact of this problem is discussed in [46][49], where a complete analysis about the violation of the main GR functioning requirements is presented. The GR decision statistics requires an estimation of the noise variance $\sigma_{\eta}^{2}$ using the reference noise $\eta_{i}[k]$ at the AF output.

Under the hypothesis $\mathscr{H}_{1}$, the signal at the GR PF output, see Fig. 1, can be defined as

$$
x_{i}[k]=s_{i}[k]+\zeta_{i}[k],
$$

where $\zeta_{i}[k]$ is the noise at the PF output and

$$
s_{i}[k]=h_{i}[k] s[k],
$$

where $h_{i}[k]$ are the channel coefficients. Under the hypothesis $\mathscr{H}_{0}$ and for all $i$ and $k$, the process $x_{i}[k]=$ $\zeta_{i}[k]$ at the PF output is subjected to the complex Gaussian distribution and can be considered as the i.i.d. process.

In the ideal case, we can think that the signal at the GR AF output is the reference noise $\eta_{i}[k]$ with the same statistical parameters as the noise $\zeta_{i}[k]$. In practice, there is a difference between the statistical parameters of the noise $\eta_{i}[k]$ and $\zeta_{i}[k]$. How this difference impacts on the GR detection performance is discussed in detail in [39, Chapter 7, pp. 631-695] and in [50] and [51],

The decision statistics at the GR output presented in [40, Chapter 3] and [41] is extended for the case of antenna array when an adoption of multiple antennas and antenna arrays is effective to mitigate the negative attenuation and fading effects. The GR decision statistics can be presented in the following form:

$$
\begin{aligned}
& T_{G R}(\mathbf{X})=\sum_{k=0}^{N-1} \sum_{i=1}^{M} 2 x_{i}[k] s_{i}^{m}[k] \\
& -\sum_{k=0}^{N-1} \sum_{i=1}^{M} x_{i}^{2}[k]+\sum_{k=0}^{N-1} \sum_{i=1}^{M} \eta_{i}^{2}[k] \underset{\mathscr{H}_{0}}{\underset{\mathcal{F}_{1}}{>}} T H R_{G R},
\end{aligned}
$$

where

$$
\mathbf{X}=[\mathbf{x}(0), \ldots, \mathbf{x}(N-1)]
$$

is the vector of the random process at the GR PF output and $T H R_{G R}$ is the GR detection threshold.

Under the hypotheses $\mathscr{H}_{1}$ and $\mathscr{H}_{0}$ when the amplitude of the transmitted signal is equal to the amplitude of the model signal, $s_{i}^{m}[k]=s_{i}[k]$ i.e., $\mu=1$, the GR decision statistics $T_{G D}(\mathbf{X})$ takes the following form in the statistical sense, respectively

$$
\left\{\begin{array}{l}
\mathscr{H}_{1}: T_{G D}(\mathbf{X})=\sum_{k=0}^{N-1} \sum_{i=1}^{M}\left\{s_{i}^{2}[k]+\eta_{i}^{2}[k]-\zeta_{i}^{2}[k]\right\} ; \\
\mathscr{H}_{0}: T_{G D}(\mathbf{X})=\sum_{k=0}^{N-1} \sum_{i=1}^{M}\left\{\eta_{i}^{2}[k]-\zeta_{i}^{2}[k]\right\} .
\end{array}\right.
$$

In (7) the term $\sum_{k=0}^{N-1} \sum_{i=1}^{M} s_{i}^{2}[k]=E_{s}$ corresponds to the average transmitted signal energy, and the term $\sum_{k=0}^{N-1} \sum_{i=1}^{M} \eta_{i}^{2}[k]-\sum_{k=0}^{N-1} \sum_{i=1}^{M} \zeta_{i}^{2}[k]$ is the background noise at the GR output. The GR output background noise is a difference between the noise power at the GR PF and GR AF outputs. Practical implementation of the GR decision statistics requires an estimation of the noise variance $\sigma_{\eta}^{2}$ using the reference noise $\eta_{i}[k]$ at the AF output.

\section{System Model}

Consider the synchronous CDMA system accommodating $K$ users. Let $x(t)$ denote the received signal for a certain bit interval, $s_{k}(t)$ is the $k$-th user's transmitted signal, $w(t)$ is the additive white Gaussian noise (AWGN). The equivalent baseband received signal can be presented in the following form:

$$
x(t)=\sum_{k=1}^{K} s_{k}(t)+w(t)=\sum_{k=1}^{K} A_{k} b_{k} a_{k}(t)+w(t), t \in[0, T]
$$

where $A_{k}$ is the $k$-th user's amplitude, $b_{k}$ is the $k$-th user's data bit, $a_{k}[t]$ denotes its signature waveform, and $T$ is the bit period. The signature waveform can be expressed as

$$
a_{k}(t)=\sum_{i=0}^{N-1} a_{k, i} \Pi_{T_{c}}\left(t-i T_{c}\right)
$$

where $a_{k, i} \in\{1 / \sqrt{N},-1 / \sqrt{N}\}$ is the binary spreading chip sequence for user $k, N$ is the processing gain, $\Pi_{T_{c}}$ is the rectangular pulse waveform with support $T_{c}$ and unit amplitude, $T_{c}$ is the chip period. 
The first stage of the generalized receiver with the parallel interference cancellation is the conventional bank of generalized receivers. The output can be presented in the following form

$$
\begin{aligned}
& y_{k}=\int_{0}^{T}\left[2 x(t) a_{k}^{m}(t)-x^{2}(t)\right] d t \\
& =A_{k} b_{k}+\sum_{j \neq k} A_{k} b_{k} \rho_{j k}+\xi_{k},
\end{aligned}
$$

where

$$
\rho_{j k}=\int_{0}^{T} a_{j}(t) a_{k}(t) d t
$$

is the coefficient of correlation and

$$
\xi_{k}=\int_{0}^{T}\left[\eta_{k}^{2}(t)-\zeta_{k}^{2}(t)\right] d t
$$

is the background noise forming at the generalized receiver output after dispreading (see Section 2). As it was discussed in Section 2, the GR CD noise component and the GR ED random component are cancelled in the statistical sense if the main functioning condition of the generalized receiver (2) is satisfied (for simplicity of analysis we assume that $\mu \rightarrow 1$ ).

It can be seen that the output metric in (10) consists of three components: the desired signal, the first term; the MAI, the second term; and the background noise $\xi_{k}$ of the generalized receiver, the third term. The conventional generalized receiver makes a decision based on the statistics $y_{k}$ given in (10). Thus, MAI is treated as another noise source. When the number of users is high, MAI will seriously degrade the system performance. A parallel interference cancellation, being a multiuser detection scheme, is proposed to alleviate this problem. Let $\hat{x}_{k}(t)$ be the interference-subtracted signal for user $k$ given by

$$
\hat{x}_{k}(t)=x(t)-\sum_{j \neq k} \hat{s}_{j}(t),
$$

where $\hat{s}_{j}(t)$ is the regenerated signal for user $j$. For the soft-decision parallel interference cancellation this signal is obtained by

$$
\hat{s}_{j}(t)=y_{j} a_{j}(t) .
$$

Thus, the output signal in the second stage is then

$$
z_{k}=\int_{0}^{T}\left[2 \hat{x}_{k}(t) a_{k}^{m}(t)-\hat{x}_{k}^{2}(t)\right] d t .
$$

Finally, the symbol data is detected as $\hat{b}_{k}=\operatorname{sgn}\left(z_{k}\right)$.

In principle, the interference cancellation procedure in (13)-(15) can be repeated with multiple stages to obtain better performance. It is apparent from (10) and (14) that the regenerated signal is noi-sy. Thus, fully cancelling the regenerated interferen-ce may not yield the best results. One solution to this problem is to partially cancel the interference. This idea is implemented by modifying (13) in the following form

$$
\hat{x}_{k}(t)=x(t)-\sum_{j \neq k} C_{j k} \hat{s}_{j}(t)
$$

The constants $C_{j k}$ are called the partial cancellation factors for the user $k$ and their amplitudes should reflect the fidelity of the interference estimate. The structure of the generalized receiver with three stages of partial soft-decision parallel interference cancellation with three users is presented in Fig. 2.

Generally, the $K \times(K-1)$ partial cancellation factors are needed for a two-stage partial parallel interference cancellation. It is apparent that the computational complexity of the partial parallel interference cancellation is high when the number of users is large on the order of $O\left(K^{2}\right)$. Two simplified structures, whose complexities are on the order of $O(K)$, were investigated in [51]. The first one corresponds to the case for that $C_{j k}=C_{j}$ in (16). In this case, all regenerated signals are first weighted and then summed. Thus, each regenerated interference signal in (16) has the individual partial cancellation factor and the signal to be estimated is a function of all partial cancellation factors. We call this structure the coupled structure.

The other structure is one, in which $C_{j k}=C_{k}$. In this case, all regenerated signals are summed first and then weighted. Thus, there is one partial cancellation factor for the signal to be estimated. We thus call this structure the decoupled structure. A thorough discussion of both structures is not available in the literature. Optimal partial cancellation factors have only derived for the coupled structure under equal power scenarios [52], [53]. In what follows, we focus our attention on the generalized receiver with the two-stage partial soft-decision parallel interference cancellation, which has the decoupled structure. Primary simulation results in Section 6 demonstrate that both parallel interference cancellation structures with the optimal partial cancellation factors have the similar performance. 


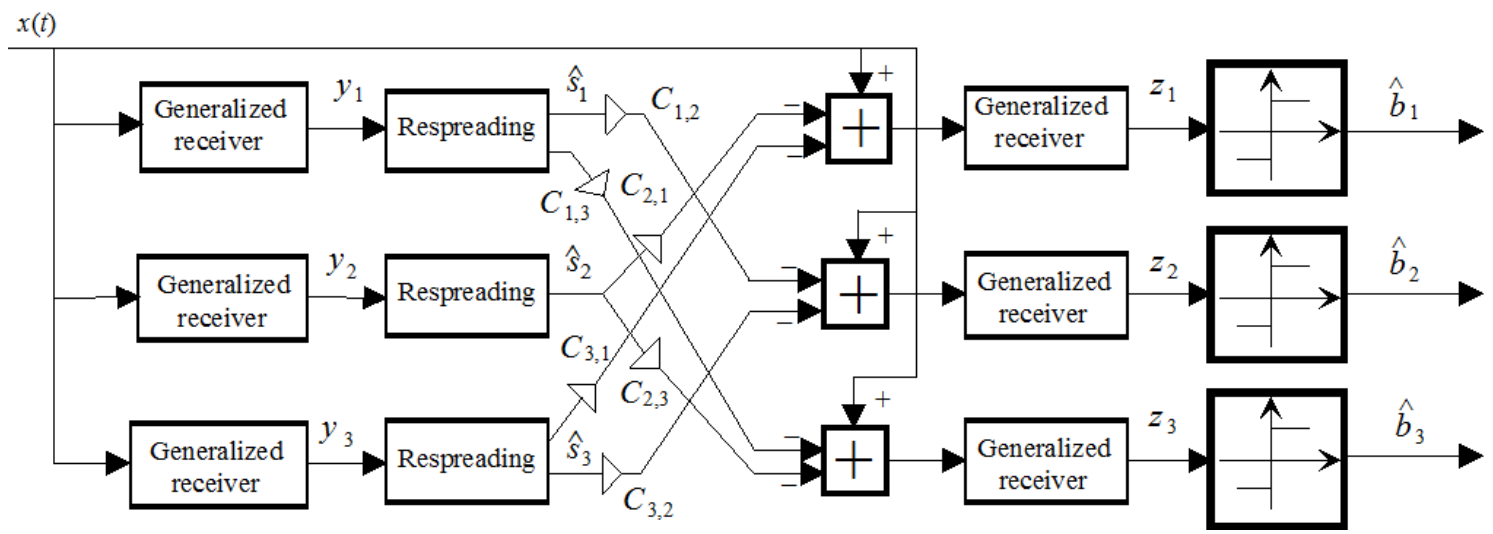

Fig. 2. Generalized receiver structure with the partial soft-decision parallel interference cancellation.

\section{Optimal Partial Cancellation Fac- tors for Additive White Gaussian Noise Channels}

In this section, we derive the optimal partial cancellation factors for the two-stage partial soft-decision parallel interference cancellation under the additive white Gaussian noise channel. For simplicity of description, we only give the results associated with synchronous transmission. Periodic and aperiodic spreading codes are both considered.

\subsection{Periodic code scenario}

Assuming perfect chip synchronization, we first sample the received continuous-time signal in (8) with the period $T_{c}$. Let

$$
\mathbf{x}=\left[x(0), x\left(T_{c}\right), \ldots, x\left((N-1) T_{c}\right]^{T}\right.
$$

be the received signal sample vector;

$$
\mathbf{a}_{k}=\left[a_{k, 0}, a_{k, 1}, \ldots, a_{k, N-1}\right]^{T}
$$

be the $k$-th user's spreading sequence vector, and

$$
\mathbf{w}=\left[w(0), w\left(T_{c}\right), \ldots, w\left((N-1) T_{c}\right]^{T}\right.
$$

be the noise sample vector. Based on (8), we have

$$
\mathbf{x}=\sum_{k} A_{k} b_{k} \mathbf{a}_{\mathbf{k}}+\mathbf{w}
$$

Thus, we can obtain the generalized receiver output process in the following form

$$
\mathbf{y}_{k}=2 \mathbf{x}^{T} \mathbf{a}_{k}-\mathbf{x} \mathbf{x}^{T}=A_{k} b_{k}+\sum_{j \neq k} A_{j} b_{j} \mathbf{a}_{j}^{T} \mathbf{a}_{k}+\xi_{k}
$$

Note that $\mathbf{a}_{j}^{T} \mathbf{a}_{k}$ is the discrete version of the correlation coefficient $\rho_{j k}$ shown in (11) and $\xi_{k}$ is the discrete -time version of the background noise (12) of the generalized receiver. Thus (21) can be rewritten in the following scalar form

$$
y_{k}=A_{k} b_{k}+\sum_{j \neq k} A_{k} b_{k} \rho_{j k}+\xi_{k} \text {. }
$$

For the second stage of the partial soft-decision interference cancellation with the decoupled structure of the generalized receiver the regenerated signal for the user $k$ takes the following form

$$
\hat{\mathbf{x}}_{k}=\mathbf{x}-C_{k} \sum_{j \neq k} \hat{\mathbf{s}}_{j},
$$

where $\hat{\mathbf{s}}_{j}=y_{j} \mathbf{a}_{j}$. The second stage output is then

$$
\begin{gathered}
z_{k}=\hat{\mathbf{x}}_{k}^{T} \mathbf{a}_{k}=y_{k}-C_{k} \sum_{j \neq k} y_{j} \rho_{j k} \\
=A_{k} b_{k}+\sum_{j \neq k} A_{j} b_{j} \rho_{j k}+\xi_{k} \\
-C_{k} \sum_{j \neq k}\left\lfloor A_{j} b_{j}+\sum_{m \neq j} A_{m} b_{m} \rho_{m j}+\xi_{j}\right\rfloor \rho_{j k} \\
=A_{k} b_{k}\left\lfloor 1-C_{k} \sum_{j \neq k} \rho_{j k}^{2}\right\rfloor+\xi_{k}-C_{k} \sum_{j \neq k} \xi_{j} \rho_{j k} \\
+\sum_{j \neq k} A_{j} b_{j}\left\lfloor\rho_{j k}-C_{k} \rho_{j k}-C_{k} \sum_{m \neq j, k} \rho_{j m} \rho_{m k}\right\rfloor .
\end{gathered}
$$

The bit error probability for the user $k$ denoted as $P_{\text {error }}\left(z_{k}\right)$ can be written as

$$
\begin{gathered}
P_{\text {error }}\left(z_{k}\right)=0.5\left[P\left(z_{k} \mid b_{k}=1\right)+P\left(z_{k} \mid b_{k}=-1\right)\right] \\
=P\left(z_{k} \mid b_{k}=1\right) .
\end{gathered}
$$

In (25) we assume that the occurrence probabilities for $b_{k}=1$ and $b_{k}=-1$ are equal, and the probabilities of error at $b_{k}=1$ and $b_{k}=-1$ are also equal. As we can see, there are three terms in (24). The first term corresponds to the desired user bit. If we let 
$b_{k}=1$, it is the deterministic value. The second term corresponds to the noise interference, which is Gaussian distributed. The third term corresponds to the interference from other users and each interference is subjected to the binomial distribution. Note that the correlation coefficients in (24) are small and the CDMA wireless communication systems are usually operated in low signal-to-noise $(S N R)$ environments. The variance of the third term is then much smaller than that of the second term. Thus, we can assume that $z_{k}$ conditioned on $b_{k}=1$ is subjected to the Gaussian distribution. The probability of error takes the following form:

$$
P_{\text {error }}\left(z_{k}\right)=Q\left(\sqrt{\frac{\mathscr{M}_{k}}{\mathscr{V}_{k}}}\right),
$$

where $Q\{\cdot\}$ is the $Q$-function and the mean and variance can be determined in the following form

$$
\begin{gathered}
\mathscr{M}_{k}=\left\{E\left[z_{k} \mid b_{k}=1\right]\right\}^{2} ; \\
\mathscr{V}_{k}=E\left(z_{k}^{2}\right)-\mathcal{M}_{k} .
\end{gathered}
$$

Note that the expectations in (27) and (28) are operated on interfering user bits and noise. Let

and

$$
E\left(\mathbf{w} \mathbf{w}^{T}\right)=\sigma_{w}^{2} \mathbf{I}_{N}
$$

$$
S N R_{j}=A_{j}^{2} / \sigma_{w}^{2} .
$$

Evaluating (27), we obtain

where

$$
\mathscr{M}_{k}=A_{k}^{2}\left(1-C_{k} \Lambda_{k}\right)^{2},
$$

$$
\Lambda_{k}=\sum_{j \neq k} \rho_{j k}^{2} .
$$

Similarly, we obtain the variance as

$$
\mathcal{V}_{k}=\sigma_{w}^{2}\left(\Omega_{1, k} C_{k}^{2}-2 \Omega_{2, k} C_{k}+\Omega_{3, k}\right),
$$

where the coefficients of $\mathscr{V}_{k}$ are represented by

$$
\begin{gathered}
\Omega_{1, k}=\sum_{j \neq k} S N R_{j}\left[\rho_{j k}+\sum_{m \neq j, k} \rho_{j m} \rho_{m k}\right]^{2} \\
+\sum_{j \neq k}\left\lfloor\rho_{j k}^{2}+\sum_{m \neq j, k} \rho_{j m} \rho_{m k} \rho_{j k}\right\rfloor ; \\
\Omega_{2, k}=\sum_{j \neq k} S N R_{j}^{2}\left\lfloor\rho_{j k}^{2}+\sum_{m \neq j, k} \rho_{j m} \rho_{m k} \rho_{j k}\right\rfloor+\sum_{j \neq k} \rho_{j k}^{2} ; \\
\Omega_{3, k}=\sum_{j \neq k} S N R_{j}^{2} \rho_{j k}^{2}+1 .
\end{gathered}
$$

The optimal partial cancellation factors for the user $k$ can be determined as

$$
\begin{gathered}
C_{k}^{o p t}=\underset{C_{k}}{\operatorname{argmax}}\left\{\mathscr{M}_{k} / \mathscr{V}_{k}\right\} \\
=\left\{C_{k}^{o p t}: \mathscr{V}_{k} \frac{d \mathscr{M}_{k}}{d C_{k}}-\mathcal{M}_{k} \frac{d \mathscr{V}_{k}}{d C_{k}}=0\right\} .
\end{gathered}
$$

Substituting (31) and (33) into (37) and simplifying the result, we have the following equation:

$$
\begin{gathered}
\left(1-C_{k}^{o p t} \Lambda_{k}\right)\left[C_{k}^{o p t}\left(\Omega_{1, k}-\Lambda_{1, k} \Omega_{2, k}\right)\right. \\
\left.+\Lambda_{k} \Omega_{3, k}-\Omega_{2, k}\right]=0 .
\end{gathered}
$$

We have two possible solutions now. The first solution for the first parenthesis is trivial since it makes the squared mean value $\mathcal{M}_{k}$ in (31) equal to zero. The optimum partial cancellation factor takes the following form

$$
C_{k}^{o p t}=\frac{\Omega_{2, k}-\Omega_{3, k} \Lambda_{k}}{\Omega_{1, k}-\Omega_{2, k} \Lambda_{k}} .
$$

We also derive the optimal partial cancellation factors for the asynchronous CDMA wireless communication system. The results are summarized in Appendix 1. In what follows, we discuss some special cases to give better understanding of characteristics of the optimal partial cancellation factor. Let the correlations between any two user spreading codes be equal, i.e. $\rho_{j k}=\rho$ at $j \neq k$, and the power control be perfect, i.e. $A_{k}=A$ and $S N R_{k}=S N R$. The optimal partial cancellation factor can then be expressed as

$$
C_{k}^{o p t}=\frac{S N R}{1+S N R[1+\rho(K-2)]} .
$$

As we can see from (40), the optimal partial cancellation factor is smaller when $\rho$ or $K$ is larger, because when the correlations between user codes are higher and the number of users is larger, the MAI is larger, and the regenerated signal is unreliable. As a result, the partial cancellation factor should be smaller. Also, when the user power is larger or the noise is smaller, the $S N R$ is the higher, the optimal partial cancellation factor is the larger. If we assume that the noise is much smaller than the signal power, i.e. $S N R>>1$, the optimal partial cancellation factor can be further simplified to

$$
C_{k}^{o p t}=\frac{1}{1+\rho(K-2)]} .
$$

Now the optimal partial cancellation factor is independent of the transmission signal power. The $B E R$ performance would also be saturated in this in- 
terference-limited region. From (40) we can also see that when the noise power is large, $S N R<<1$, the optimal partial cancellation factor tends to be small, $C_{k} \rightarrow 0$. Note that the effect of the processing gain $N$ is reflected in the receiving $S N R$. If $N$ is larger, the receiving $S N R$ will become smaller.

\subsection{Aperiodic code scenario}

In commercial CDMA wireless communication systems the users' spreading codes are often modulated with another code having a very long period. As far as the received signal is concerned, the spreading code is not periodic. In other words, there will be many possible spreading codes for each user. If we use the result derived above, we then have to calculate the optimum partial cancellation factors for each possible code and the computational complexity will become very high. Since the period of the modulating code is usually very long, we can treat the code chips as independent random variables and approximate the correlation coefficients $\rho_{j k}$, as the Gaussian random variable. As a result, the expectations in (27) and (28) can be further operated on $\rho_{j k}$. This greatly simplifies the optimal partial cancellation factor evaluation. We now rewrite (26) in the following form

$$
P_{\text {error }}\left(z_{k}\right)=Q\left(\sqrt{\frac{E_{\mathscr{L}}\left\{\mathcal{M}_{k}^{(l)}\right\}}{E_{\mathscr{L}}\left\{\mathcal{V}_{k}^{(l)}\right\}}}\right),
$$

where $E_{\mathscr{L}}\{\}$ denotes the expectation operator over the spreading code set $\mathscr{L}$ and $\mathscr{N}_{k}^{(l)}$ and $\mathscr{V}_{k}^{(l)}$ are the expected squared mean and variance of $z_{k}$, respecttively, given the $l$-th possible code in $\mathscr{L}$. Letting

$$
I_{k}=\sum_{j \neq k} S N R_{j}^{2}
$$

and considering $\rho_{j k}$ as the Gaussian random variable, and evaluating (27) and (28), we obtain

$$
E_{\mathscr{L}}\left\{M_{k}^{(l)}\right\}=A_{k}^{2}\left[1-C_{k} E_{\mathscr{L}}\left\{\Lambda_{k}^{(l)}\right\}\right]^{2},
$$

where

$$
E_{\mathscr{L}}\left\{\Lambda_{k}^{(l)}\right\}=\frac{K-1}{N}
$$

and

$$
\begin{gathered}
E_{\mathscr{L}}\left\{\mathscr{V}_{k}^{(l)}\right\} \\
=\sigma_{w}^{2}\left[E_{\mathscr{L}}\left\{\Omega_{1, k}^{(l)}\right\} C_{k}^{2}-2 E_{\mathscr{L}}\left\{\Omega_{2, k}^{(l)}\right\} C_{k}+E_{\mathscr{L}}\left\{\Omega_{3, k}^{(l)}\right\}\right],
\end{gathered}
$$

where

$$
E_{\mathscr{L}}\left\{\Omega_{1, k}^{(l)}\right\}
$$

$$
\begin{gathered}
=I_{k}\left(\frac{1}{N}+\frac{3(K-2)}{N^{2}}+\frac{(K-2)(K-3)}{N^{3}}\right) \\
+\frac{K-1}{N}+\frac{(K-1)(K-2)}{N^{2}} ; \\
E_{\mathscr{L}}\left\{\Omega_{2, k}^{(l)}\right\}=I_{k}\left(\frac{1}{N}+\frac{K-2}{N^{2}}\right)+\frac{K-1}{N} ; \\
E_{\mathscr{L}}\left\{\Omega_{3, k}^{(l)}\right\}=\frac{I_{k}}{N}+1 .
\end{gathered}
$$

In the above expressions, the notation $X^{(l)}$ denotes the value $X$ given the $l$-th possible spreading code in $\mathscr{L}$. Equation (37) can be rewritten in the following form:

$$
\begin{aligned}
& C_{k}^{o p t}=\underset{C_{k}}{\operatorname{argmax}}\left\{\frac{E_{\mathscr{L}}\left\{\mathcal{M}_{k}^{(l)}\right\}}{E_{\mathscr{L}}\left\{\mathscr{V}_{k}^{(l)}\right\}}\right\} \\
= & \left\{C_{k}^{o p t}: E_{\mathscr{L}}\left\{\mathscr{V}_{k}^{(l)}\right\} \frac{d E_{\mathscr{L}}\left\{\mathscr{M}_{k}^{(l)}\right\}}{d C_{k}}\right. \\
- & \left.E_{\mathscr{L}}\left\{\mathcal{M}_{k}^{(l)}\right\} \frac{d E_{\mathscr{L}}\left\{\mathscr{V}_{k}^{(l)}\right\}}{d C_{k}}=0\right\} .
\end{aligned}
$$

Substituting (44)-(49) into (50) and simplifying the result, we finally obtain

$$
C_{k}^{o p t}=\frac{E_{\mathscr{L}}\left\{\Omega_{2, k}^{(l)}\right\}-E_{\mathscr{L}}\left\{\Omega_{3, k}^{(l)}\right\} E_{\mathscr{L}}\left\{\Lambda_{k}^{(l)}\right\}}{E_{\mathscr{L}}\left\{\Omega_{1, k}^{(l)}\right\}-E_{\mathscr{L}}\left\{\Omega_{2, k}^{(l)}\right\} E_{\mathscr{L}}\left\{\Lambda_{k}^{(l)}\right\}} .
$$

As we can see, Eq. (51) only involves Eq. (45) and

Eqs. (47)-(49) and these expressions are easy to work with. We further consider the case, in which the noise is small, $I_{k}>K$. Equation (51) can be simplified to

$$
C_{k}^{o p t}=\frac{N}{N+2 K-4} .
$$

This result is remarkably simple. We only require $N$ and $K$ to calculate the optimal partial cancellation factors. This will be useful in real-world applications.

\section{Optimal Partial Cancellation Fac- tors for Multipath Channels}

\subsection{Periodic code scenario}

Let the transfer function for the user $k$ 's channel be

$$
H_{k}(z)=\sum_{i=1}^{L} h_{k, i} z^{-\tau_{k, i}} .
$$

As we can see from (53), the number of paths is $L$ and the gain and delay for the $i$-th channel path are 
$h_{k, i}$ and $\tau_{k, i}$, respectively. We use two vectors to represent these parameters

and

$$
\mathbf{t}_{k}=\left[\tau_{k, 1}, \tau_{k, 2}, \ldots, \tau_{k, L}\right]^{T}
$$

$$
\mathbf{h}_{k}=\left[h_{k, 1}, h_{k, 2}, \ldots, h_{k, L}\right]^{T} .
$$

Let $\tau_{k, 1} \leq \tau_{k, 2} \leq \cdots \leq \tau_{k, L}$ and the channel power is normalized, i.e. $\sum_{k, i} h_{k, i}^{2}=1$. Without loss of generality, we may assume that $\tau_{k, 1}=0$ for each user and $L$ is the maximum possible number of paths. When a user's path number, say $L^{\prime}$, is less than $L$, we can let all elements in $\tau_{k, i}$ and $h_{k, i}$ be zero for $L^{\prime}+1 \leq i \leq L$. We may also assume that the maximum delay is much smaller than the processing gain $N$ [54]. Before our formulation, we first define the $(2 N-1) \times L$ composite signature matrix $\mathbf{S}_{k}$ in the following form

$$
\mathbf{S}_{k}=\left[\widetilde{\mathbf{a}}_{k, 1}, \widetilde{\mathbf{a}}_{k, 2}, \ldots, \widetilde{\mathbf{a}}_{k, L}\right],
$$

where $\widetilde{\mathbf{a}}_{k, i}$ is the vector containing $i$-th delayed spreading code for the user $k$. It is defined as

$$
\widetilde{\mathbf{a}}_{k, i}=[\overbrace{0, \ldots, 0,}^{\tau_{k, i}}, \mathbf{a}_{k}^{T}, \overbrace{0, \ldots, 0}^{N-\tau_{k, i}-1}]^{T} .
$$

Since a multipath channel is involved, the current received bit signal will be interfered by previous bit signals. As mentioned above, the maximum path delay is much smaller than the processing gain. The interference will not be severe and for simplicity, we may ignore this effect. Let

$$
\mathbf{f}_{k}=\mathbf{S}_{k} \mathbf{h}_{k} .
$$

As that in (20), we can obtain the received signal vector as

$$
\mathbf{x}=\sum_{k} A_{k} b_{k} \mathbf{f}_{k}+\mathbf{w} .
$$

To have better results, we use the maximum ra-tio rake combining scheme in the generalized recei-ver. Let

$$
\left\{\begin{array}{l}
\rho_{j k}=\mathbf{f}_{j}^{T} \mathbf{f}_{k} ; \\
\rho_{k}=\rho_{k k} ; .
\end{array}\right.
$$

The output of the generalized receiver takes the following form

$$
\begin{gathered}
y_{k}=2 \mathbf{x}^{T} \mathbf{f}_{k}-\mathbf{x} \mathbf{x}^{T}=A_{k} b_{k} \mathbf{f}_{k}^{T} \mathbf{f}_{k}+\sum_{j \neq k} A_{j} b_{j} \mathbf{f}_{j}^{T} \mathbf{f}_{k}+\xi_{k} \\
=A_{k} b_{k} \rho_{k}+\sum_{j \neq k} A_{j} b_{j} \rho_{j k}+\xi_{k} .
\end{gathered}
$$

This result is similar to that in (21) except that $\rho_{j k}$ is replaced by $\rho_{j k}$. For the second stage of the partial soft-decision parallel interference cancellation, the regenerated signal takes the following form

$$
\hat{\mathbf{x}}_{k}=\mathbf{x}-C_{k} \sum_{j \neq k} \hat{\mathbf{s}}_{j}=\mathbf{x}-C_{k} \sum_{j \neq k} y_{j} \mathbf{f}_{j} .
$$

We then have the output signal for the second stage as

$$
\begin{gathered}
z_{k}=2 \mathbf{x}_{k}^{T} \mathbf{f}_{k}-\mathbf{x}_{k} \mathbf{x}_{k}^{T} \\
=A_{k} b_{k}\left\lfloor\rho_{k}-C_{k} \sum_{j \neq k} \rho_{j, k}^{2}\right\rfloor+v_{k}-C_{k} \sum_{j \neq k} v_{j} \rho_{j k} \\
+\sum_{j \neq k} A_{j} b_{j}\left\lfloor\rho_{j k}-C_{k} \rho_{j k}-C_{k} \sum_{m \neq j, k} \rho_{j m} \rho_{m k}\right\rfloor .
\end{gathered}
$$

As previously, we assume that $z_{k}$ is approximated by the Gaussian probability distribution density, the interfering bits and noise are random, and parameters $N, K, \mathbf{t}_{k}, \mathbf{h}_{k}, S N R_{k}, \rho_{j k}$ are known beforehand. Thus, the output probability of error is expressed as in (26) where the squared mean for $z_{k}$, similar to that of (31), is obtained from (27) and (63) in the following form

$$
M_{k}=A_{k}^{2}\left(\rho_{k}-C_{k} \Gamma_{k}\right)^{2},
$$

where

$$
\Gamma_{k}=\sum_{j \neq k} \rho_{j k}^{2}
$$

and the variance is obtained from (28) and (63) as

$$
\mathscr{V}_{k}=\sigma_{w}^{2}\left(\Xi_{1, k} C_{k}^{2}-2 \Xi_{2, k} C_{k}+\Xi_{3, k}\right),
$$

where

$$
\begin{gathered}
\Xi_{1, k}=\sum_{j \neq k} S N R_{j}^{2}\left[\rho_{j k} \rho_{j}+\sum_{m \neq j, k} \rho_{j m} \rho_{m k}\right]^{2} \\
+\sum_{j \neq k}\left\lfloor\rho_{j k}^{2} \rho_{j}+\sum_{m \neq j, k} \rho_{j m} \rho_{m k} \rho_{j k}\right\rfloor ; \\
\Xi_{2, k}=\sum_{j \neq k} S N R_{j}^{2}\left\lfloor\rho_{j k}^{2} \rho_{j}+\sum_{m \neq j, k} \rho_{j m} \rho_{m k} \rho_{j k}\right\rfloor+\sum_{j \neq k} \rho_{j k}^{2} ; \\
\Xi_{3, k}=\sum_{j \neq k} S N R_{j}^{2} \rho_{j k}^{2}+\rho_{j k} .
\end{gathered}
$$

The optimal partial cancellation factor derivation for the multipath channels is similar to that in (37). Substituting (64) and (66) into (37), we then obtain 


$$
C_{k}^{o p t}=\frac{\rho_{k} \Xi_{2, k}-\Xi_{3, k} \Gamma_{k}}{\rho_{k} \Xi_{1, k}-\Xi_{2, k} \Gamma_{k}}
$$

\subsection{Aperiodic code scenario}

If aperiodic codes are utilized, the correlation coefficients $\rho_{j k}$ can be considered as the Gaussian random variables. Using the method in Section 4 we can obtain the corresponding optimal partial cancellation factors. From (64) we have the expected squared mean in the following form

$$
\begin{gathered}
E_{\mathscr{L}}\left\{\mathcal{M}_{k}^{(l)} \rho_{k}^{(l)}\right\}=A_{k}^{2}\left[E_{\mathscr{L}}\left\{\rho_{k}^{(l)}-C_{k} E_{\mathscr{L}}\left\{\Gamma_{k}^{(l)}\right\}\right]^{2}\right. \\
=A_{k}^{2}\left[1-C_{k} E_{\mathscr{L}}\left\{\Gamma_{k}^{(l)}\right\}\right]^{2}
\end{gathered}
$$

and the variance takes the form

$$
\begin{gathered}
E_{\mathscr{L}}\left\{\mathscr{V}_{k}^{(l)}\right\} \\
=\sigma_{w}^{2}\left[E_{\mathscr{L}}\left\{\Xi_{1, k}^{(l)}\right\} C_{k}^{2}-2 E_{\mathscr{L}}\left\{\Xi_{2, k}^{(l)}\right\} C_{k}+E_{\mathscr{L}}\left\{\Xi_{3, k}^{(l)}\right\}\right] .
\end{gathered}
$$

Comparing (71) and (72) with (44)-(46), we see that the optimal partial cancellation factor here is similar to that in (50). We then have the partial cancellation factor in the following form

$$
C_{k}^{o p t}=\frac{E_{\mathscr{L}}\left\{\Xi_{2, k}^{(l)}\right\}-E_{\mathscr{L}}\left\{\Xi_{3, k}^{(l)}\right\} E_{\mathscr{L}}\left\{\Gamma_{k}^{(l)}\right\}}{E_{\mathscr{L}}\left\{\Xi_{1, k}^{(l)}\right\}-E_{\mathscr{L}}\left\{\Xi_{2, k}^{(l)}\right\} E_{\mathscr{L}}\left\{\Gamma_{k}^{(l)}\right\}} .
$$

Unlike that in the additive white Gaussian noise channel, the result for the aperiodic code scenario is more difficult to obtain because there are more correlation terms in (65) and (67)-(69) to work with. Before evaluating expectation terms in (73), we define some functions as follows:

$$
\begin{gathered}
h_{j k}(p, q)=h_{j, p} h_{k, q} ; \\
\tau_{j k}(p, q)=\tau_{j, p}-\tau_{k, q} ; \\
\chi_{j k}(p, q)=\widetilde{\mathbf{a}}_{j, p}^{T} \widetilde{\mathbf{a}}_{k, q} .
\end{gathered}
$$

Thus, (74)-(76) define some relative figures between the $p$-th channel path of the $j$-th user and $q$-th channel path of the $k$-th user. The notation $h_{j k}(p, q)$ denotes the path gain product, $\tau_{j k}(p, q)$ is the relative path delay, and $\chi_{j k}(p, q)$ is the code correlation with the relative delay $\tau_{j k}(p, q)$. Expanding (67)-(69), we have seven expectation terms to evaluate. For purpose of illustration, we show how to evaluate the first term, $E_{\mathscr{L}}\left\{\rho_{j k}^{2}\right\}$ here. By definition, the correlation coefficient $\rho_{j k}$ can be presented in the following form

$$
\begin{gathered}
\rho_{j k}=\mathbf{f}_{j}^{T} \mathbf{f}_{k}=\left(\sum_{p=1}^{L} \widetilde{\mathbf{a}}_{j, p} h_{j, p}\right)^{T}\left(\sum_{q=1}^{L} \widetilde{\mathbf{a}}_{k, q} h_{k, q}\right) \\
=\sum_{p=1}^{L} \sum_{q=1}^{L} h_{j, p} h_{k, q} \widetilde{\mathbf{a}}_{j, p}^{T} \mathbf{a}_{k, q}=\sum_{p=1}^{L} \sum_{q=1}^{L} h_{j k}(p, q) \chi_{j k}(p, q) .
\end{gathered}
$$

The mathematical expectation of the correlation coefficient $\rho_{j k}$ over all possible codes is then obtained in the following form:

$$
\begin{aligned}
E_{\mathscr{L}}\left\{\rho_{j k}^{2}\right\}= & E\left\{\sum_{p_{1}=1}^{L} \sum_{q_{1}=1}^{L} \sum_{p_{2}=1}^{L} \sum_{q_{2}=1}^{L} h_{j k}\left(p_{1}, q_{1}\right) \chi_{j k}\left(p_{1}, q_{1}\right)\right. \\
& \left.\times h_{j k}\left(p_{2}, q_{2}\right) \chi_{j k}\left(p_{2}, q_{2}\right)\right\} \\
= & \sum_{p_{1}=1}^{L} \sum_{q_{1}=1}^{L} \sum_{p_{2}=1}^{L} \sum_{q_{2}=1}^{L} h_{j k}\left(p_{1}, q_{1}\right) h_{j k}\left(p_{2}, q_{2}\right) \\
& \times E\left\{\chi_{j k}\left(p_{1}, q_{1}\right) \chi_{j k}\left(p_{2}, q_{2}\right)\right\}
\end{aligned}
$$

Let

$$
\mathscr{F}_{j k}\left(p_{1}, q_{1}, p_{2}, q_{2}\right)=N^{2} E\left\{\chi_{j k}\left(p_{1}, q_{1}\right) \chi_{j k}\left(p_{2}, q_{2}\right)\right\} .
$$

The coefficient $N^{2}$ in (79) is only the normalization constant. Since the spreading codes are seen as random, only when $\tau_{j k}\left(p_{1}, q_{1}\right)$ is equal to $\tau_{j k}\left(p_{2}, q_{2}\right)$ will $\mathscr{F}_{j k}(\cdot)$ be nonzero. Consider a specific set of $\left\{p_{1}\right.$, $\left.q_{1}, p_{2}, q_{2}\right\}$ such that

$$
\tau_{j k}\left(p_{1}, q_{1}\right)=\tau_{j k}\left(p_{2}, q_{2}\right)=\tau, \tau \geq 0 .
$$

We then have

$\mathscr{F}_{j k}\left(p_{1}, q_{1}, p_{2}, q_{2}\right)=N^{2} \sum_{\nu=0}^{N-\tau-1} E\left\{a_{j, v+\tau}^{2} a_{k, v}^{2}\right\}=N-\tau$.

For $\tau<0$, we have the same result except that the sign of $\tau$ in (81) is plus. We then conclude that the function $\mathscr{F}_{j k}(\cdot)$ in (79) takes the following form:

$$
\begin{gathered}
\mathscr{F}_{j k}\left(p_{1}, q_{1}, p_{2}, q_{2}\right) \\
= \begin{cases}N-|\tau|, & \text { if } \tau_{j k}\left(p_{1}, q_{1}\right)=\tau_{j k}\left(p_{2}, q_{2}\right)=\tau, \\
0, & \text { otherwise } .\end{cases}
\end{gathered}
$$

Using (78), (79), and (82), we can evaluate $E_{\mathscr{L}}\left\{\rho_{j k}^{2}\right\}$ in (67)-(69). The general formulations for the other six mathematical expectations and variances are summarized in Appendix 2. 
We now provide a simple example to show the multipath effect on the optimal partial cancellation factors. Let $\forall k, \alpha^{2}+\beta^{2}=1$, then

$$
\begin{gathered}
\left\{\begin{array}{l}
\mathbf{t}_{k}=[0, D]^{T} ; \\
\mathbf{h}_{k}=[\alpha, \beta]^{T} ;
\end{array}\right. \\
\left\{\begin{array}{l}
\mathscr{O}_{a}=(N-D) \alpha^{2} \beta^{2} ; \\
\mathscr{O}_{b}=(N-2 D) \alpha^{4} \beta^{4} ;
\end{array}\right.
\end{gathered}
$$

In this case we can write

$$
\begin{gathered}
E_{\mathscr{L}}\left\{\Gamma_{k}^{(l)}\right\}=E_{\mathscr{L}}\left\{\Lambda_{k}^{(l)}\right\}+\frac{2 \mathscr{C}_{a}(K-1)}{N^{2}} ; \\
E_{\mathscr{L}}\left\{\Xi_{1, k}^{(l)}\right\}=E_{\mathscr{L}}\left\{\Omega_{1, k}^{(l)}\right\}+2 \mathscr{C}_{a}\left\{\frac { I _ { k } } { N ^ { 4 } } \left[N^{2}+10 N+4 \mathscr{C}_{a}\right.\right. \\
\left.+2(K-2)\left(4 N+3 K+\mathscr{E}_{a}+1\right]+\frac{K-1}{N^{3}}(N+3 K-2)\right\} \\
+\mathscr{E}_{b}\left\{\frac{I_{k} K}{N^{4}}+6 K-12\right\}+\frac{I_{k}}{N^{4}}(6 N-10 D) \alpha^{4} \beta^{4} ;(86) \\
E_{\mathscr{L}}\left\{\Xi_{2, k}^{(l)}\right\}=E_{\mathscr{L}}\left\{\Omega_{2, k}^{(l)}\right\} \\
+2 \mathscr{C}_{a}\left[\frac{I_{k}}{N^{3}}(N+3 K-2)+\frac{K-1}{N^{2}}\right] ; \\
E_{\mathscr{L}}\left\{\Xi_{3, k}^{(l)}\right\}=E_{\mathscr{L}}\left\{\Omega_{3, k}^{(l)}\right\}+2 \mathscr{I}_{a} \frac{I_{k}}{N^{2}} .
\end{gathered}
$$

Note that the first terms in (85)-(88) are those in (45) and (47)-(49) which correspond to the optimal partial cancellation factors in the additive white Gaussian noise channel of CDMA wireless communication system. Other terms are due to the multipath channel effect. It is evident to see that if $\beta=0, \mathscr{C}_{a}=$ $\mathscr{C}_{b}=0$ and the metrics above are then degenerated to (45) and (47)-(49).

\section{Simulation Results}

\subsection{Performance comparison for various partial parallel interference cancellation structures}

In this subsection, we provide the simulation results to verify the validity of our derived partial cancellation factors. Before we do that, we present some comparison results to justify the parallel interference cancellation structure we considered. First, we compare the performance of the partial soft-decision parallel interference cancellation and that of the partial hard-decision parallel cancellation. We used periodic codes of length 31 as spreading codes.

Let $E_{b} / \mathcal{N}_{0}=8 \mathrm{~dB}$, where $\sigma_{w}^{2}=0.5 \mathcal{N}_{0}$, and assume a perfect power control scenario. It is straightfor- ward to see that in the perfect power control case, the optimal partial cancellation factors are equal for the coupled and decoupled structures. Figure 3 demonstrates the $B E R$ performance versus the number of users $K$. Here, the optimal partial cancellation factors for the partial hard-decision parallel interference cancellation were determined empirically (trial and error with a resolution of 0.01). Surprisingly, we found that the optimal partial soft-decision parallel interference cancellation outperformed the optimal partial hard-decision parallel interference cancellation. This result differs from the result given in [36], [53]-[56] where the full soft-decision partial interference cancellation was found to be inferior to the full hard-decision parallel interference cancellation. Additionally we made the performance comparison between the generalized receiver and matched filter. We see that generalized receiver outperforms the matched filter receiver by performance.

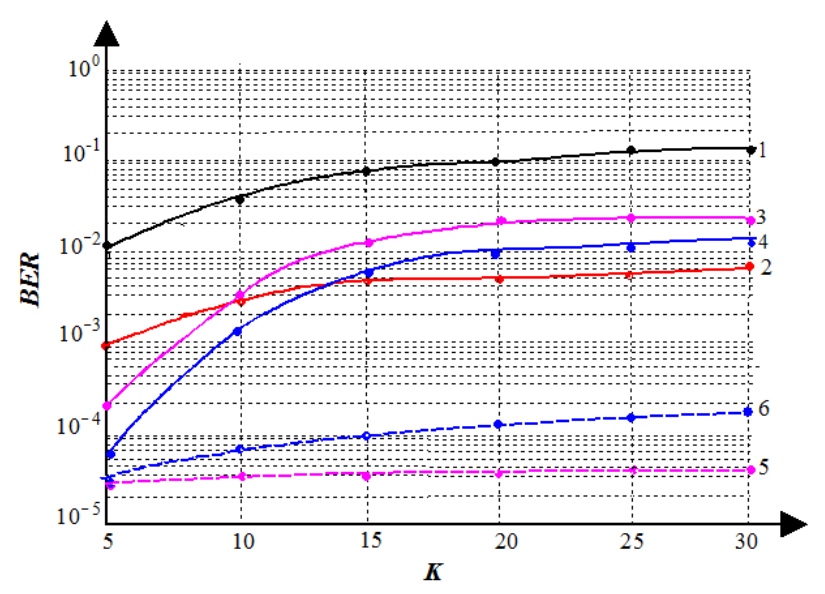

Fig. 3. Performance comparison for hard- and soft-decision parallel interference cancellation $\left(N=31, p=N^{-0.5}\right.$, $\left.E_{b} / \mathcal{N}_{0}=8 \mathrm{~dB}\right)$. Optimal partial cancellation factors for the partial hard-decision parallel interference cancellation were obtained by trial and error and those for the soft-decision parallel interference cancellation were obtained from (39): 1-conventional matched filter; 2-conventional generalized receiver; 3 -full soft-decision parallel interference cancellation; 4-full hard-decision parallel interference cancellation; 5- partial soft-decision parallel interference cancellation; 6- partial hard-decision parallel interference cancellation.

In the second set of simulation, we made a comparison between the performance of the coupled and decoupled structures using the partial soft-decision parallel interference cancellation. As mentioned above, the optimal partial cancellation factors are equal for both structures under perfect power control. Thus, we compared their performance in an imperfect power control scenario. The optimal partial can- 
cellation factors for the coupled structure were determined empirically.

Let the number of users be three and the spreading code be a periodic of length 31 . We assume that the third user has the fixed $S N R$, i.e. $E_{b} / \mathcal{N}_{0}=8 \mathrm{~dB}$, and the other two users have variable $S N R$, for example, the first user has $E_{b} / \mathfrak{N}_{0}-\Delta S N R=8-\Delta S N R$ $\mathrm{dB}$ and the second user has $E_{b} / \mathfrak{N}_{0}-2 \Delta S N R=8-$ $2 \triangle S N R \mathrm{~dB}$, respectively. Figure 4 represents the $B E R$ performance as a function of $\triangle S N R$ for these three structures. As we can see, both structures, coupled and decoupled, possess the similar BER performance.

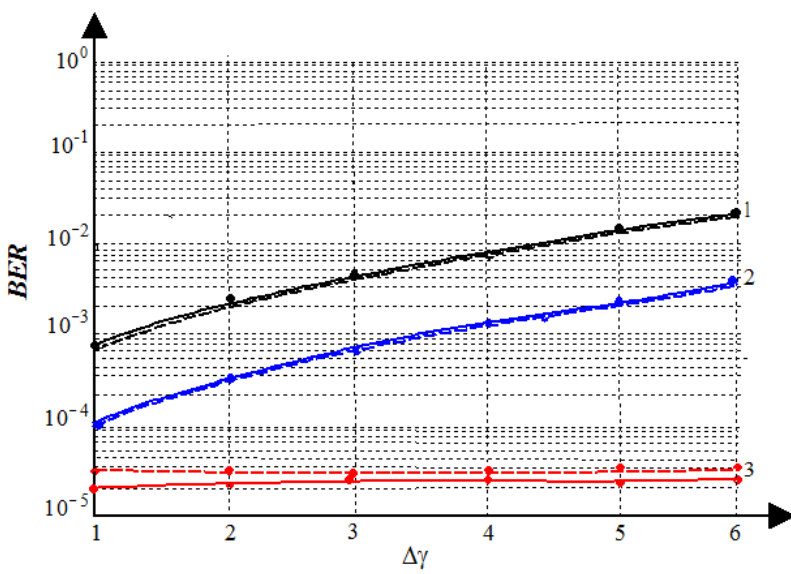

Fig. 4. Performance comparison for the coupled (solid line) and decoupled (dashed line) structures (three users with $E_{b} / \mathcal{N}_{0}=8-2 \Delta S N R, 8-\Delta S N R$, and $8 \mathrm{~dB}$ ). Optimal partial cancellation factors for the coupled structure were obtained by trial and error, and those for the decoupled structure were obtained from (51); 1-user \#1; 2-user \#2; 3- user \#3.

\subsection{Validity of derived partial cancellation factors}

In this subsection, we report simulation results demonstrating the accuracy of theoretical solutions carried out in previous sections for the optimal partial soft-decision parallel interference cancellation. A two-stage decoupled partial parallel interference cancellation is considered. For the simulations conducted, we used the Gold codes for periodic code systems and random codes for aperiodic code systems. Figure 5 gives the empirical and theoretical $B E R$ performances for the generalized receiver with optimal partial soft-decision parallel interference cancellation under the aperiodic code scenario. This figure shows the validity of the Gaussian approximation used in our derivation. As we can see, when the number of users is smaller and the $S N R=E_{b} / \mathfrak{e N}_{0}^{\circ}$ is higher, the Gaussian approximation is less valid.
Figure 6 demonstrates the optimal partial cancellation factors in (39) and the empirical optimal partial cancellation factors as a function of the number of users. The channel here is the asynchronous additive white Gaussian noise channel. The spreading codes are periodic and $E_{b} / \mathcal{N}_{0}=8 \mathrm{~dB}$ for each user. We can see from this figure that the theoretical optimal partial cancellation factors are very close to the empirical ones in all cases.

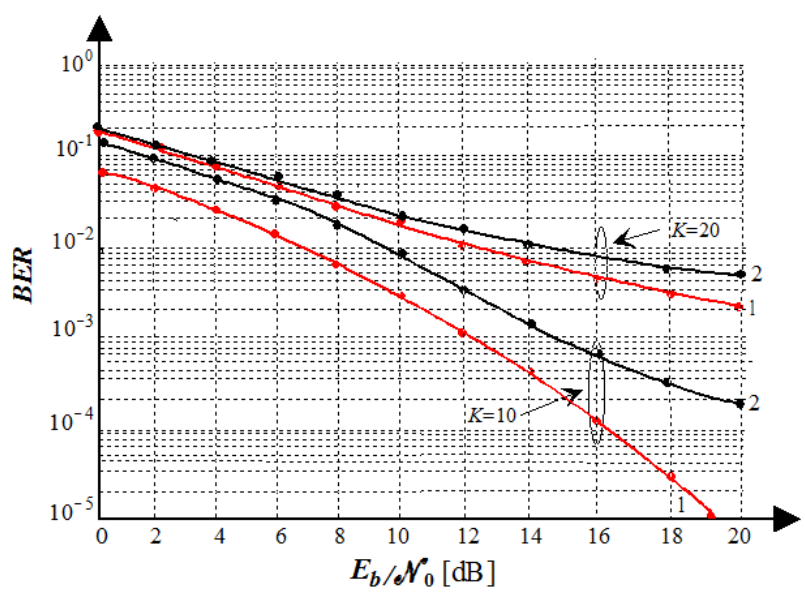

Fig. 5. BER performance of the generalized receiver with the partial soft-decision parallel interference cancellation as a function of $E_{b} / \mathcal{N}_{0}$ (aperiodic AWGN channels and perfect power control): 1-theoretical curve; 2-empirical curve.

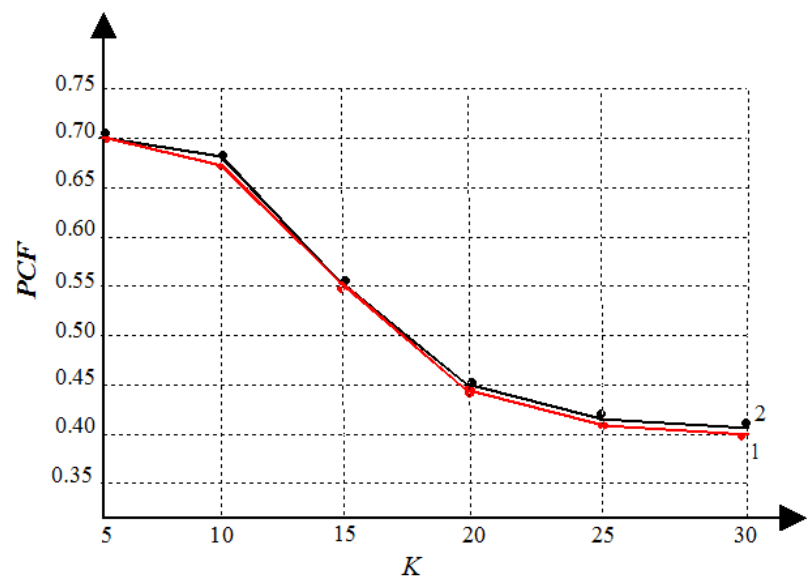

Fig. 6. Optimal partial cancellation factor (PCF) versus the number of users (Gold codes, asynchronous AWGN channels, $E_{b} / \mathcal{N}_{0}=8 \mathrm{~dB}$, and perfect power control): 1theoretical curve; 2- empirical curve.

We then consider the optimal partial cancellation factors for the multipath channel. The assumed multipath channel is the two-ray channel with the transfer function $H_{k}(z)=0.762+0.648 z^{-2}$ for all users. Theoretical optimal partial cancellation factors derived in (73) are compared with empirical partial can- 
cellation factors and the results are presented in Fig. 7. We can observe that the theoretical results are also matched with the empirical ones satisfactorily. Note that when the number of users is smaller, the theoretical values are less accurate. This is because when the number of users is small, the Gaussian approximation in (42) is less valid. This is also consistent with the results observed in Fig. 5.

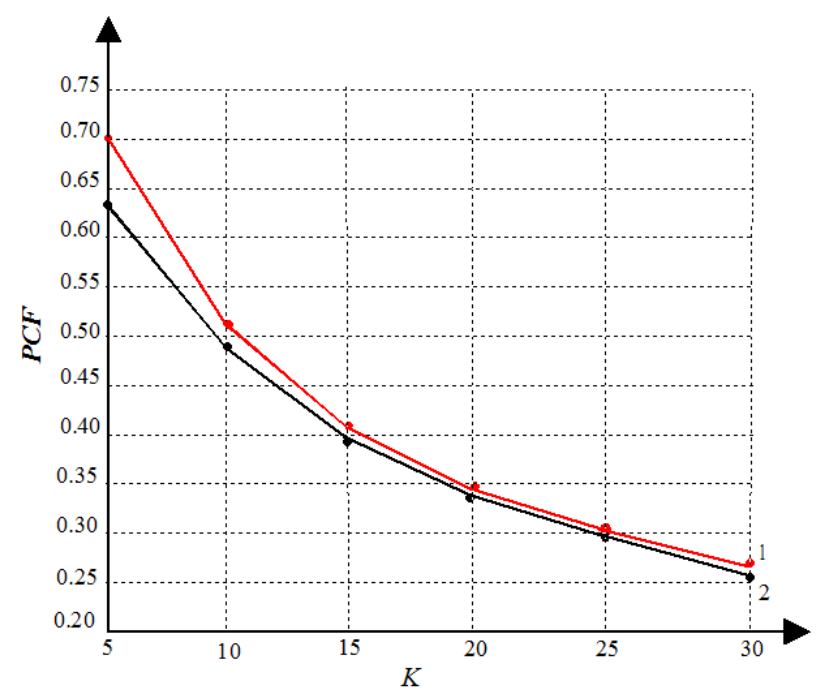

Fig. 7. Optimal partial cancellation factor (PCF) versus the number of users (a periodic codes, multipath channels, $E_{b} / \mathcal{N}_{0}=10 \mathrm{~dB}$, and perfect power control): 1-theoretical curve; 2 -empirical curve.

\subsection{BER performance comparison}

In what follows, we report the $B E R$ performance for the matched filter and generalized receivers with soft-decision parallel interference cancellation. Figure 8 demonstrates comparative analysis of performances between the conventional matched filter receiver and generalized one in the case of the optimal two-stage partial soft-decision parallel interference cancellation, namely, the two-stage full soft-decision parallel interference cancellation and the three stage full soft-decision parallel interference cancellation. From Fig. 8 we see that BER performance of the generalized receiver outperforms the matched filter performance. The spreading codes are periodic and the channel is the asynchronous channel with the additive white Gaussian noise, also, $S N R=E_{b}$ $/ \mathcal{N}_{0}=10 \mathrm{~dB}$ and perfect power control is assumed. From Fig. 8, we can see that the optimal two-stage partial soft-decision parallel interference cancellation possesses better performance in comparison with other cases.

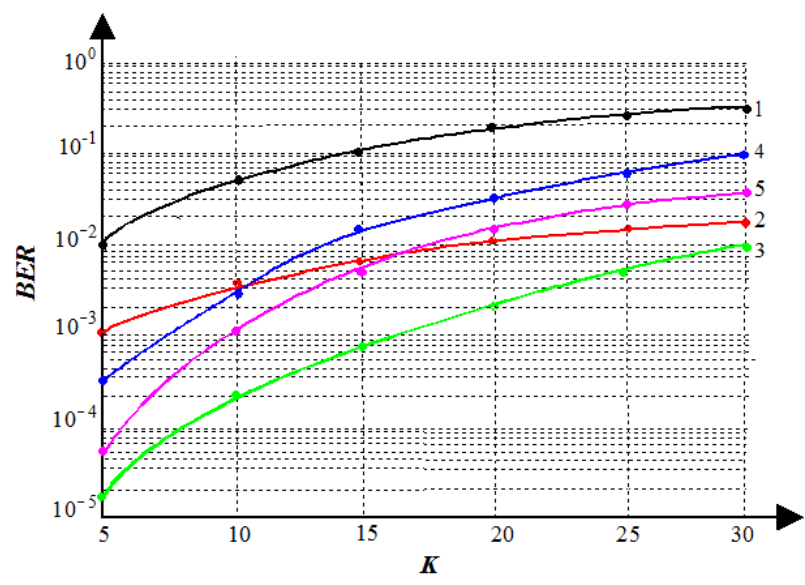

Fig. 8. BER performance versus the number of users (Gold codes, asynchronous AWGN channels, $E_{b} / \mathcal{N}_{0}=$ $10 \mathrm{~dB}$, and perfect power control): 1- the matched filter receiver; 2- the generalized receiver; 3 - the generalized receiver with 2 - the stage partial soft-decision interference cancellation; 4- the generalized receiver with the 2-stage full soft-decision interference cancellation; 5- the generalized receiver with 3 -stage full soft-decision interference cancellation.

The performance of the generalized receiver with the two-stage and three-stage soft-decision parallel interference cancellation is better than the performance of the conventional matched filter receiver. The performances of the generalized receiver with the two-stage and three-stage soft-decision parallel interference cancellation are worse in comparison with ones of the conventional generalized receiver when the number of users is large. The optimal two-stage partial soft-decision parallel interference cancellation always is better than the conventional generalized receiver performance.

Finally, Fig. 9 demonstrates the performance comparison for the generalized receiver and Rake detector considered in the previous sections for the case of the multipath channel. The simulation setup is identical to that in the previous cases except that the spreading code is a periodic. The partial cancellation factors for the optimal two-stage partial soft-decision parallel interference cancellation have been calculated using (73). As in the case of channel with the additive white Gaussian noise, the performance of the generalized receiver with the optimal two-stage partial soft-decision parallel interference cancellation outperforms the performance of other types of the generalized detectors. Additionally, we see a great superiority of employment of the generalized receiver in comparison with the Rake one in the considered DS-CDMA wireless communication systems. 


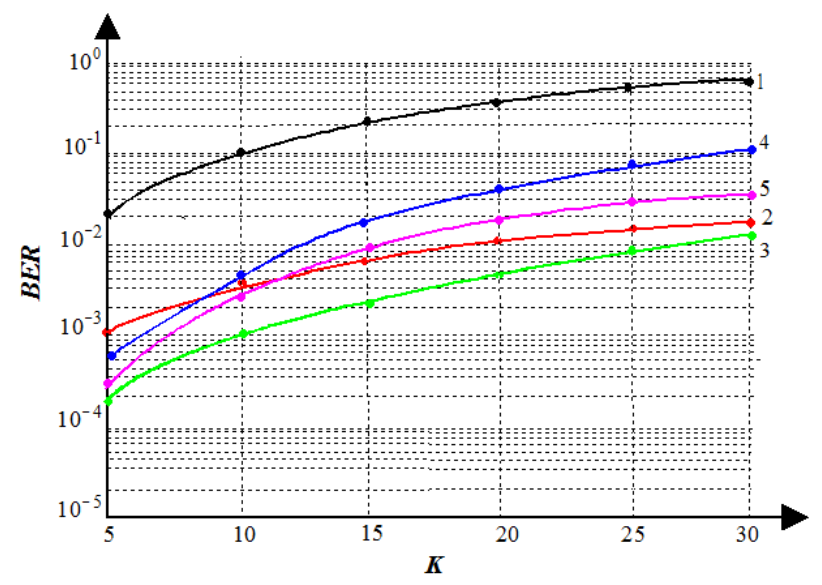

Fig. 9. BER performance versus the number of users (a periodic spreading codes, asynchronous multipath channels, $E_{b} / \mathcal{N}_{0}=10 \mathrm{~dB}$, and perfect power control): 1-the Rake receiver; 2-the generalized receiver; 3-the generalized receiver with 2 -stage partial soft-decision interference cancellation; 4-the generalized receiver with 2-stage full soft-decision interference cancellation; 5-the generalized receiver with 3 -stage full soft-decision interference cancellation.

\subsection{Effect of imperfect parameter estimation}

In the optimal partial cancellation factor formulation we assume that the required parameters are perfectly known. In practice, this may not be always possible. Some parameters will have to be estimated for timevarying channels which may introduce errors. The main parameters we need to know are the channel responses and the noise variance. Once the channel responses are known, $A_{k}, \rho_{j k}, S N R_{k}$ can be calculated accordingly. We model the channel estimation error as follows. Let $g_{k, i}=A_{k} h_{k, i}$ be the $i$-th path channel of the user $k$, and $g_{k, i}^{\prime}=g_{k, i}+\Delta g_{k, i}$, where $g_{k, i}^{\prime}$ is the estimated channel response, $g_{k, i}$ is the actual response, and $\Delta g_{k, i}$ is the Gaussian random variable denoting the estimation error.

We first let the noise variance be exactly known and varied the channel estimation error. The performance impact is shown in Fig. 10. The result corresponds to the case in which the user number is six, the spreading code is a periodic, the channel is the multipath channel, and $S N R=E_{b} / \mathcal{N}_{0}=10 \mathrm{~dB}$. In Fig. $10, \sigma_{g}^{2}$ is the variance of $\Delta g_{k, i}$ that is the same for $\forall k, i$. Since the conventional generalized receiver and the generalized receiver with the full soft-decision parallel interference cancellation do not rely on channel information, the channel estimation error has no influence on their performance. The variation of the BER performance in Fig. 10 are due to the random data used in different runs. Additionally, we see a great superiority of employment of the generalized receiver in comparison with the Rake one in the considered DS-CDMA wireless communication systems.

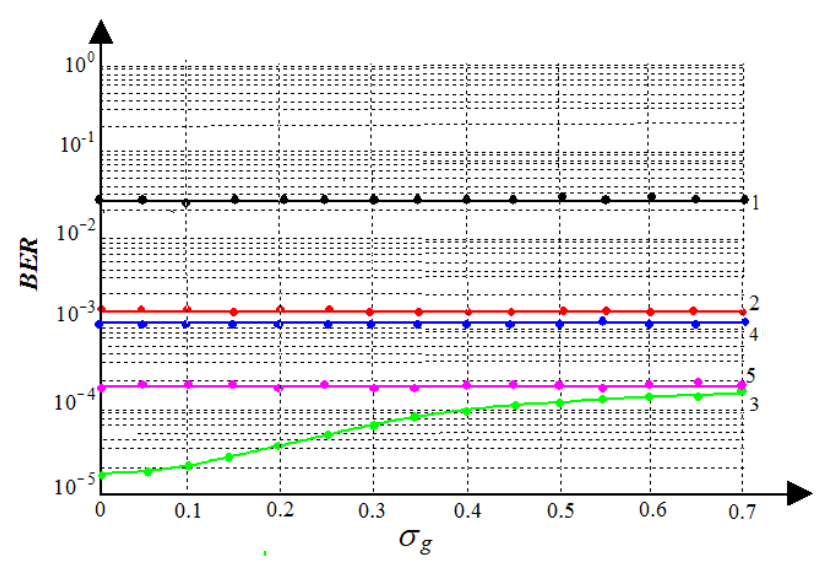

Fig. 10. BER performance with channel estimation error (a periodic spreading codes, multipath channels, $K=6$ $E_{b} / \mathcal{N}_{0}=10 \mathrm{~dB}$, and perfect power control): 1-the Rake receiver; 2-the generalized receiver; 3-the generalized receiver with 2-stage partial soft-decision interference cancellation; 4-the generalized receiver with 2-stage full softdecision interference cancellation; 5-the generalized receiver with 3-stage full soft-decision interference cancellation.

As we can see, the performance of the generalized receiver with the partial soft-decision parallel interference cancellation is not affected until $\sigma_{g}^{2}=$ 0.09 . Note that the magnitude of the main path is 0.762. Thus, the estimation error is quite high in this case. The second case we consider is the noise variance estimation error. The simulation setup is identical to the previous one. We let the channel responses be known and varied the noise variance from 0.1 $\times \sigma_{w}^{2}$ to $10 \times \sigma_{w}^{2}$, where $\sigma_{w}^{2}$ is the actual noise variance. We find that the optimal soft-decision parallel interference cancellation performance is almost unaffected. Thus, we conclude that the optimal partial soft-decision parallel interference cancellation has good immunity to parameter estimation errors.

\section{Conclusions}

In DS-CDMA wireless communication systems, MAI is considered as the main factor in the system performance degradation. Among multiuser detection schemes, the generalized receiver with the parallel interference cancellation is considered as a simple and effective approach. It has been shown that the performance of the generalized receiver with the parallel interference cancellation can be further improved if interference is not fully cancelled. The perfor- 
mance of the generalized receiver with the partial parallel interference cancellation depends totally on the partial cancellation factors. Thus, how to determine the partial cancellation factors optimally is then of great concern.

In the present paper, we have considered the generalized receiver with the two-stage decoupled partial soft-decision interference cancellation and derived a set of closed-form solutions for the case of optimal partial cancellation factors. These partial cancellation factors are useful for periodic and aperiodic spreading codes in channels with the additive white Gaussian noise and those in multipath channels. Simulation results show that the derived optimal partial cancellation factors agree closely with empirical optimal partial cancellation factors. The performance of the generalized receiver with the optimal twostage partial soft-decision parallel interference cancellation outperforms the performance of the conventional generalized receiver when the number of users is more than 10 and has superiority in comparison with the generalized receiver with the two-stage full soft-decision parallel interference cancellation and even with the tree-stage full soft-decision parallel interference cancellation.

We have also shown that performance of the generalized receiver with the derived partial cancellation factors is not sensitive to parameter estimation errors. The optimal partial cancellation factors for aperiodic spreading code systems in AWGN channel have a simple expression. This will be a great advantage for real world applications since the optimal partial cancellation factors can be determined efficiently online in a time-varying environment.

In the present paper, we are mainly concerned with BPSK modulation. Note that the same result can be extended to accommodate QAM modulation. In this case, however, we have to take the interference between in phase quadrature components into account. It turns out that for the in phase or quadrature component of one user, we may treat the number of interfering users as $2 K-1$.

\section{Appendix 1:Periodic Code System Op- timal PCFs for Asynchronous AWGN \\ Channels}

Let $b_{k, j}$ denote the $i$-th bit for the $k$-th user and $\tau_{k}$ is the user delay. Then the received signal for asynchronous channels can then be represented in the following form

$$
x(t)=\sum_{k} \sum_{i} b_{k, i} a_{k}\left(t-i T-\tau_{k}\right) \Pi_{T}\left(t-i T-\tau_{k}\right)+w(t) .
$$

We further define the relative delay between the users $j$ and $k$ as $\tau_{j, k}=\tau_{j}-\tau_{k}$, and the cross-correlation functions are given by

$$
\rho_{j k}\left(\tau_{j k}\right)= \begin{cases}\int_{T+\tau_{j k}}^{T} a_{j}\left(t-\tau_{j k}-T\right) a_{k}(t) d t, & \tau_{j k}<0, \\ \int_{\tau_{j k}}^{T} a_{j}\left(t-\tau_{j k}\right) a_{k}(t) d t, & \tau_{j k} \geq 0\end{cases}
$$

and

$$
\hat{\rho}_{j k}\left(\tau_{j k}\right)= \begin{cases}\int_{0}^{T+\tau_{j k}} a_{j}\left(t-\tau_{j k}\right) a_{k}(t) d t, & \tau_{j k}<0, \\ \int_{\tau_{j k}}^{T} a_{j}\left(t-\tau_{j k}\right) a_{k}(t) d t, & \tau_{j k} \geq 0 .\end{cases}
$$

For simplicity, we use $\rho_{j k}$ and $\hat{\rho}_{j k}$ instead of $\rho_{j k}\left(\tau_{j k}\right)$ and $\hat{\rho}_{j k}\left(\tau_{j k}\right)$ in the sequel. The process at the output of the generalized receiver for the $k$-th user's $i$-the bit is obtained in the following form

$$
\begin{gathered}
y_{k, i}=\int_{i T+\tau_{k}}^{(i+1) T+\tau_{k}}\left[2 x(t) a_{k}\left(t-\tau_{k}\right)-x(t) x\left(t-\tau_{k}\right)\right. \\
\left.+\eta_{k}(t) \eta_{k}\left(t-\tau_{k}\right)\right] d t \\
=A_{j} b_{k, i}+\sum_{j \neq k} A_{j}\left(b_{j, i-l, l k} \hat{\rho}_{j k}+b_{j, i-l, l j} \rho_{j k}\right)+\xi_{k, i},
\end{gathered}
$$

where the delay index and noise term are expressed as $l_{j k}$ and $\xi_{k i}$. They are defined as

$$
l_{j k}= \begin{cases}1, & \tau_{j k} \geq 0 \\ 0, & \text { otherwise }\end{cases}
$$

and (see Fig. 1 and (7))

$$
\xi_{k i}=\int_{i T+\tau_{k}}^{(i+1) T+\tau_{k}}\left[\eta_{k}(t) \eta_{k}\left(t-\tau_{k}\right)-\zeta_{k}(t) \zeta_{k}\left(t-\tau_{k}\right)\right] d t
$$

The regenerated received signal using the partial soft-decision parallel interference cancellation is given by

$$
\begin{gathered}
\hat{x}_{k}(t)=x(t) \\
-C_{k} \sum_{j \neq k} \sum_{i} y_{j, i} a_{j}\left(t-i T-\tau_{j}\right) \Pi_{T}\left(t-i T-\tau_{j}\right) .
\end{gathered}
$$

Thus, the second stage output can be presented in the following form

$$
z_{k, i}=\int_{i T+\tau_{k}}^{(i+1) T+\tau_{k}}\left[2 \hat{x}(t) a_{k}\left(t-\tau_{k}\right)-\hat{x}(t) \hat{x}\left(t-\tau_{k}\right)\right.
$$




$$
\begin{gathered}
\left.+\eta_{k}(t) \eta_{k}\left(t-\tau_{k}\right)\right] d t=y_{k, i} \\
-C_{k} \sum_{j \neq k}\left(y_{j, i-l_{j k}} \hat{\rho}_{j k}+y_{j, i-l_{j k}+1} \rho_{j k}\right) \\
=A_{k} b_{k, i}+\sum_{j \neq k} A_{j}\left(b_{j, i-l_{j k}} \hat{\rho}_{j k}+b_{j, i-l_{j k}+1} \rho_{j k}\right)+\xi_{k, i} \\
-C_{k} \sum_{j \neq k}\left\{A_{j}\left(b_{j, i-l_{j k}} \hat{\rho}_{j k}+b_{j, i-l_{j k}+1} \rho_{j k}\right)\right. \\
+\sum_{m \neq j} A_{m}\left(b_{m, i-l_{m j}-l_{j k}} \hat{\rho}_{m j} \hat{\rho}_{j k}+b_{m, i-l_{m j}-l_{j k}+1} \rho_{m j} \hat{\rho}_{j k}\right. \\
\left.+b_{m, i-l_{m j}-l_{j k+1}} \hat{\rho}_{m j} \rho_{j k}+b_{m, i-l_{m j}-l_{j k}+2} \rho_{m j} \rho_{j k}\right) \\
\left.+\xi_{j, i-l_{j k}} \hat{\rho}_{j k}+\xi_{j, i-l_{j k}+1} \rho_{j k}\right\}
\end{gathered}
$$

Without loss of generality, we may assume that $\tau_{j} \geq$ $\tau_{k}, j \neq k$. Then $l_{j k}=1, \forall j$ and the result can be simplified to

$$
\begin{array}{r}
z_{k, i}=A_{k} b_{k, i}\left\{1-C_{k} \sum_{j \neq k}\left(\hat{\rho}_{j k}^{2}+\rho_{j k}^{2}\right)\right\} \\
-A_{k} C_{k} \sum_{j \neq k}\left(b_{k, i-1}+b_{k, i+1}\right) \hat{\rho}_{j k} \rho_{j k} \\
-C_{k} \sum_{j \neq k} \sum_{m \neq j, k}^{\tau_{m} \leq \tau_{j}} A_{j} b_{j, i-2} \hat{\rho}_{j m} \hat{\rho}_{m k} \\
-C_{k} \sum_{j \neq k} \sum_{m \neq j, k}^{\tau_{m} \leq \tau_{j}} A_{j} b_{j, i+1} \rho_{j m} \rho_{m k} \\
+\sum_{j \neq k} A_{j} b_{j, i-1} \Phi_{j k}+\sum_{j \neq k} A_{j} b_{j, i} \Psi_{j k}+\xi_{k, i} \\
-C_{k} \sum_{j \neq k}\left(\xi_{j, i-l_{j k}} \hat{\rho}_{j k}+\xi_{j, i-l_{j k}+1} \rho_{j k}\right)
\end{array}
$$

where $\Phi_{j k}$ and $\Psi_{j k}$ are defined in the following form

$$
\begin{gathered}
\Phi_{j k}=\hat{\rho}_{j k}\left(1-C_{k}\right) \\
-C_{k}\left\{\sum_{m \neq j, k}^{\tau_{m}>\tau_{j}} \hat{\rho}_{j m} \hat{\rho}_{m k}+\sum_{m \neq j, k}^{\tau_{m} \leq \tau_{j}}\left(\rho_{j m} \hat{\rho}_{m k}+\hat{\rho}_{j m} \rho_{m k}\right)\right\}, \\
\Psi_{j k}=\rho_{j k}\left(1-C_{k}\right) \\
-C_{k}\left\{\sum_{m \neq j, k}^{\tau_{m} \leq \tau_{j}} \rho_{j m} \rho_{m k}+\sum_{m \neq j, k}^{\tau_{m}>\tau_{j}}\left(\rho_{j m} \hat{\rho}_{m k}+\hat{\rho}_{j m} \rho_{m k}\right)\right\} .
\end{gathered}
$$

The squared-mean for $z_{k, i}$ is obtained from (27) and (96) in the following form

$$
M_{k}=A_{k}^{2}\left(1-C_{k} \Lambda_{k}\right)^{2}
$$

where

$$
\Lambda_{k}=\sum_{j \neq k}\left(\hat{\rho}_{j k}^{2}+\rho_{j k}^{2}\right)
$$

Similarly, the variance can also be obtained as

$$
\mathcal{V}_{k}=\sigma_{w}^{2}\left(\Omega_{1, k} C_{k}^{2}-2 \Omega_{2, k} C_{k}+\Omega_{3, k}\right)
$$

where $\Omega_{i, k}, 1 \leq i \leq 3$ are defined in the following form

$$
\begin{gathered}
\Omega_{1, k}=2 S N R_{k}^{2} \sum_{j \neq k} \hat{\rho}_{j k}^{2} \rho_{j k}^{2}+\sum_{j \neq k} S N R_{j}^{2}\left(\Phi_{j k}^{2}+\Psi_{j k}^{2}\right) \\
+\left(\sum_{j \neq k} \sum_{m \leq j, k}^{\tau_{m} \leq \tau_{j}} S N R_{j}^{2} \hat{\rho}_{j m} \hat{\rho}_{m k}\right)^{2}+\left(\sum_{j \neq k} \sum_{m \leq j, k}^{\tau_{m}>\tau_{j}} S N R_{j}^{2} \rho_{j m} \rho_{m k}\right)^{2} \\
+\sum_{j \neq k} \hat{\rho}_{j k}^{2}+\rho_{j k}^{2} \\
+\sum_{j \neq k} \sum_{m \leq j, k}^{\tau_{m} \leq \tau_{j}} 2\left(\rho_{j m} \hat{\rho}_{j k} \hat{\rho}_{m k}+\rho_{j m} \rho_{j k} \rho_{m k}+\hat{\rho}_{j m} \hat{\rho}_{j k} \rho_{m k}\right) \\
+\sum_{j \neq k} \sum_{m \leq j, k}^{\tau_{m} \leq \tau_{j}}\left(\rho_{j m} \hat{\rho}_{j k} \hat{\rho}_{m k}+\rho_{j m} \rho_{j k} \rho_{m k}\right) ; \\
\Omega_{2, k}=\sum_{j \neq k} S N R_{j}^{2}\left(\hat{\rho}_{j k} \Phi_{j k}+\rho_{j k} \Psi_{j k}\right)+\sum_{j \neq k}\left(\hat{\rho}_{j k}^{2}+\rho_{j k}^{2}\right) \\
\Omega_{3, k}=\sum_{j \neq k} S N R_{j}^{2}\left(\hat{\rho}_{j k}^{2}+\rho_{j k}^{2}\right)+1 .
\end{gathered}
$$

Thus, the optimal partial cancellation factor can be obtained by substituting (100) and (102)-(104) into (39).

\section{Appendix 2: Expressions for the Expe- cted Terms in (67)-(69)}

Extending the definition in (79) we have

$$
\begin{gathered}
\mathscr{F}_{j k}\left(p_{1}, q_{1}, \ldots, p_{i}, q_{i}\right) \\
=N^{i} E\left\{\chi_{j k}\left(p_{1}, q_{1}\right), \ldots, \chi_{j k}\left(p_{i}, q_{i}\right)\right\},
\end{gathered}
$$

where $i$ is the integer. To make this expression simpler, we let $\mathbf{w}_{i}=\left\{p_{i}, q_{i}\right\}$. Then, (105) can be presented in the following form

$$
\mathscr{F}_{j k}\left(\mathbf{w}_{1}, \ldots, \mathbf{w}_{i}\right)=N^{i} E\left\{\chi_{j k}\left(\mathbf{w}_{1}\right), \ldots, \chi_{j k}\left(\mathbf{w}_{i}\right)\right\}
$$

We further omit the subscript in $\mathscr{F}(\cdot)$ and use the following notational substitution:

$$
\sum_{\mathbf{w}_{i}=0}^{L^{2}} \rightarrow \sum_{p_{i}=0}^{L} \sum_{q_{i}=0}^{L}
$$

In what follows, six expected terms are given without detailed derivation

$$
E_{\mathscr{L}}\left(\rho_{j k}^{2} \rho_{j}\right)=\frac{1}{N^{3}}
$$


$\times \sum_{\mathbf{w}_{1}=1 \mathbf{w}_{2}=1 \mathbf{w}_{4}=1}^{L^{2}} \sum^{L^{2}} \sum_{j k}^{L^{2}}\left(\mathbf{w}_{1}\right) h_{j k}\left(\mathbf{w}_{2}\right) h_{j j}\left(\mathbf{w}_{3}\right) \mathscr{F}\left(\mathbf{w}_{1}, \mathbf{w}_{2}, \mathbf{w}_{3}\right)$, if $\tau_{j k}\left(\mathbf{w}_{1}\right)=\tau_{j k}\left(\mathbf{w}_{2}\right), \tau_{j j}\left(\mathbf{w}_{3}\right)=0$,

$$
\mathscr{F}\left(\mathbf{w}_{1}, \mathbf{w}_{2}, \mathbf{w}_{3}\right)=N\left(N-\left|\tau_{j k}\left(\mathbf{w}_{1}\right)\right|\right) .
$$

Else if $\left|\tau_{j k}\left(\mathbf{w}_{1}\right)-\tau_{j k}\left(\mathbf{w}_{2}\right)\right|=\left|\tau_{j j}\left(\mathbf{w}_{3}\right)\right|$,

$$
\begin{gathered}
\mathscr{F}\left(\mathbf{w}_{1}, \mathbf{w}_{2}, \mathbf{w}_{3}\right)=N \\
-\max \left\{\left|\tau_{j k}\left(\mathbf{w}_{1}\right)\right|,\left|\tau_{j k}\left(\mathbf{w}_{2}\right)\right|,\left|\tau_{j j}\left(\mathbf{w}_{3}\right)\right|\right\} .
\end{gathered}
$$

Else

$$
\begin{gathered}
\mathscr{F}\left(\mathbf{w}_{1}, \mathbf{w}_{2}, \mathbf{w}_{3}\right)=0 \\
E_{\mathscr{L}}\left(\rho_{j k}^{2} \rho_{j m} \rho_{m k}\right)=\frac{1}{N^{3}} \\
\times \sum_{\mathbf{w}_{1}=1 \mathbf{w}_{2}=1 \mathbf{w}_{3}=1}^{L^{2}} \sum_{j k}^{L^{2}} h_{1}\left(\mathbf{w}_{1}\right) h_{j m}\left(\mathbf{w}_{2}\right) h_{m k}\left(\mathbf{w}_{3}\right) \mathscr{F}\left(\mathbf{w}_{1}, \mathbf{w}_{2}, \mathbf{w}_{3}\right) \\
\operatorname{if} \tau_{j m}\left(\mathbf{w}_{1}\right)+\tau_{m k}\left(\mathbf{w}_{2}\right)=\tau_{j k}\left(\mathbf{w}_{3}\right), \\
\mathscr{F}\left(\mathbf{w}_{1}, \mathbf{w}_{2}, \mathbf{w}_{3}\right)=N \\
-\max \left\{\left|\tau_{j k}\left(\mathbf{w}_{1}\right)\right|,\left|\tau_{j m}\left(\mathbf{w}_{2}\right)\right|,\left|\tau_{m k}\left(\mathbf{w}_{3}\right)\right|\right\} .
\end{gathered}
$$

Else

$$
\begin{gathered}
\mathscr{F}\left(\mathbf{w}_{1}, \mathbf{w}_{2}, \mathbf{w}_{3}\right)=0 . \\
E_{\mathscr{L}}\left(\rho_{j k}^{2} \rho_{j}\right)=\frac{1}{N^{4}}
\end{gathered}
$$$$
\left.\times \sum_{\mathbf{w}_{1}=1 \mathbf{w}_{2}=1 \mathbf{w}_{3}=1 \mathbf{w}_{4}=1}^{L^{2}} \sum_{j k}^{L^{2}} \sum^{2} \mathbf{w}_{1}\right) h_{j k}\left(\mathbf{w}_{2}\right) h_{j j}\left(\mathbf{w}_{3}\right) h_{j j}\left(\mathbf{w}_{4}\right)
$$$$
\times \mathscr{F}\left(\mathbf{w}_{1}, \mathbf{w}_{2}, \mathbf{w}_{3}, \mathbf{w}_{4}\right),
$$

if $\tau_{j k}\left(\mathbf{w}_{1}\right)=\tau_{j k}\left(\mathbf{w}_{2}\right), \tau_{j j}\left(\mathbf{w}_{3}\right)=\tau_{j j}\left(\mathbf{w}_{4}\right)=0$,

$$
\mathscr{F}\left(\mathbf{w}_{1}, \mathbf{w}_{2}, \mathbf{w}_{3}, \mathbf{w}_{4}\right)=N^{2}\left(N-\left|\tau_{j k}\left(\mathbf{w}_{1}\right)\right|\right) .
$$

Else if $\tau_{j k}\left(\mathbf{w}_{1}\right)=\tau_{j k}\left(\mathbf{w}_{2}\right), \tau_{j j}\left(\mathbf{w}_{3}\right)=\tau_{j j}\left(\mathbf{w}_{4}\right)$, $\mathscr{F}\left(\mathbf{w}_{1}, \mathbf{w}_{2}, \mathbf{w}_{3}, \mathbf{w}_{4}\right)=\left(N-\left|\tau_{j k}\left(\mathbf{w}_{1}\right)\right|\right)\left(N-\left|\tau_{j j}\left(\mathbf{w}_{3}\right)\right|\right)$. Else if $\left|\tau_{j k}\left(\mathbf{w}_{1}\right)-\tau_{j k}\left(\mathbf{w}_{2}\right)\right|=\tau_{j j}\left(\mathbf{w}_{3}\right), \tau_{j j}\left(\mathbf{w}_{4}\right)=0$, or $\left|\tau_{j k}\left(\mathbf{w}_{1}\right)-\tau_{j k}\left(\mathbf{w}_{2}\right)\right|=\tau_{j j}\left(\mathbf{w}_{4}\right), \tau_{j j}\left(\mathbf{w}_{3}\right)=0$,

$\mathscr{F}\left(\mathbf{w}_{1}, \mathbf{w}_{2}, \mathbf{w}_{3}, \mathbf{w}_{4}\right)=N$

$-\max \left\{\left|\tau_{j k}\left(\mathbf{w}_{1}\right)\right|,\left|\tau_{j k}\left(\mathbf{w}_{2}\right)\right|,\left|\tau_{j j}\left(\mathbf{w}_{3}\right)\right|,\left|\tau_{j j}\left(\mathbf{w}_{4}\right)\right|\right\}$.

Else if $\left|\tau_{j k}\left(\mathbf{w}_{1}\right)-\tau_{j k}\left(\mathbf{w}_{2}\right)\right|=\left|\tau_{j j}\left(\mathbf{w}_{3}\right) \pm \tau_{j j}\left(\mathbf{w}_{4}\right)\right|$,

$$
\begin{gathered}
\tau_{j k}\left(\mathbf{w}_{1}\right) \neq 0, \tau_{j k}\left(\mathbf{w}_{2}\right) \neq 0, \\
\mathscr{F}\left(\mathbf{w}_{1}, \mathbf{w}_{2}, \mathbf{w}_{3}, \mathbf{w}_{4}\right)=N \\
-\max \left\{\left|\tau_{j k}\left(\mathbf{w}_{1}\right)\right|,\left|\tau_{j k}\left(\mathbf{w}_{2}\right)\right|,\left|\tau_{j j}\left(\mathbf{w}_{3}\right)\right|,\left|\tau_{j j}\left(\mathbf{w}_{4}\right)\right|,\right. \\
\left|\tau_{j k}\left(\mathbf{w}_{1}\right)-\tau_{j k}\left(\mathbf{w}_{2}\right)\right|,\left|\tau_{j k}\left(\mathbf{w}_{1}\right) \pm \tau_{j j}\left(\mathbf{w}_{3}\right)\right|, \\
\left.\left|\tau_{j k}\left(\mathbf{w}_{1}\right) \pm \tau_{j j}\left(\mathbf{w}_{4}\right)\right|\right\} .
\end{gathered}
$$

Else if $\left|\tau_{j k}\left(\mathbf{w}_{1}\right)-\tau_{j k}\left(\mathbf{w}_{2}\right)\right|=\left|\tau_{j j}\left(\mathbf{w}_{3}\right) \pm \tau_{j j}\left(\mathbf{w}_{4}\right)\right|$,

$$
\mathscr{F}\left(\mathbf{w}_{1}, \mathbf{w}_{2}, \mathbf{w}_{3}, \mathbf{w}_{4}\right)=N
$$

$$
-\max \left\{\left|\tau_{j k}\left(\mathbf{w}_{1}\right)\right|,\left|\tau_{j k}\left(\mathbf{w}_{2}\right)\right|,\left|\tau_{j j}\left(\mathbf{w}_{3}\right)\right|,\left|\tau_{j j}\left(\mathbf{w}_{4}\right)\right|\right\} .
$$

Else

$$
\begin{gathered}
\mathscr{F}\left(\mathbf{w}_{1}, \mathbf{w}_{2}, \mathbf{w}_{3}, \mathbf{w}_{4}\right)=0 . \\
E_{\mathscr{L}}\left(\rho_{j m}^{2} \rho_{m k}^{2}\right)=\frac{1}{N^{4}} \\
\left.\times \sum_{\mathbf{w}_{1}=1 \mathbf{w}_{2}=1 \mathbf{w}_{3}=\mathbf{w}_{4}=1}^{L^{2}} \sum_{j m}^{L^{2}} \sum^{2}{ }^{L_{1}}\right) h_{j m}\left(\mathbf{w}_{2}\right) h_{m k}\left(\mathbf{w}_{3}\right) h_{m k}\left(\mathbf{w}_{4}\right) \\
\times \mathscr{F}\left(\mathbf{w}_{1}, \mathbf{w}_{2}, \mathbf{w}_{3}, \mathbf{w}_{4}\right), \\
\text { if } \tau_{j m}\left(\mathbf{w}_{1}\right)=\tau_{j m}\left(\mathbf{w}_{2}\right), \tau_{m k}\left(\mathbf{w}_{3}\right)=\tau_{m k}\left(\mathbf{w}_{4}\right), \\
\mathscr{F}\left(\mathbf{w}_{1}, \mathbf{w}_{2}, \mathbf{w}_{3}, \mathbf{w}_{4}\right)=\left(N-\left|\tau_{j m}\left(\mathbf{w}_{1}\right)\right|\right)\left(N-\left|\tau_{m k}\left(\mathbf{w}_{3}\right)\right|\right) . \\
\text { Else if }\left|\tau_{j m}\left(\mathbf{w}_{1}\right)-\tau_{j m}\left(\mathbf{w}_{2}\right)\right|=\left|\tau_{m k}\left(\mathbf{w}_{3}\right)-\tau_{m k}\left(\mathbf{w}_{4}\right)\right|, \\
\tau_{j m}\left(\mathbf{w}_{1}\right) \neq 0, \tau_{j m}\left(\mathbf{w}_{2}\right) \neq 0, \tau_{m k}\left(\mathbf{w}_{3}\right) \neq 0, \tau_{m k}\left(\mathbf{w}_{4}\right) \neq 0, \\
\mathscr{F}\left(\mathbf{w}_{1}, \mathbf{w}_{2}, \mathbf{w}_{3}, \mathbf{w}_{4}\right)=N \\
-\max \left\{\left|\tau_{j m}\left(\mathbf{w}_{1}\right)\right|,\left|\tau_{j m}\left(\mathbf{w}_{2}\right)\right|,\left|\tau_{m k}\left(\mathbf{w}_{3}\right)\right|,\left|\tau_{m k}\left(\mathbf{w}_{4}\right)\right|,\right. \\
\left|\tau_{j m}\left(\mathbf{w}_{1}\right)-\tau_{j m}\left(\mathbf{w}_{2}\right)\right|,\left|\tau_{j m}\left(\mathbf{w}_{1}\right)+\tau_{m k}\left(\mathbf{w}_{3}\right)\right|, \\
\left.\left|\tau_{j m}\left(\mathbf{w}_{1}\right)+\tau_{m k}\left(\mathbf{w}_{4}\right)\right|\right\} .
\end{gathered}
$$

Else if $\left|\tau_{j m}\left(\mathbf{w}_{1}\right)-\tau_{j m}\left(\mathbf{w}_{2}\right)\right|=\left|\tau_{m k}\left(\mathbf{w}_{3}\right)-\tau_{m k}\left(\mathbf{w}_{4}\right)\right|$,

$$
\mathscr{F}\left(\mathbf{w}_{1}, \mathbf{w}_{2}, \mathbf{w}_{3}, \mathbf{w}_{4}\right)=N
$$

$-\max \left\{\left|\tau_{j m}\left(\mathbf{w}_{1}\right)\right|,\left|\tau_{j m}\left(\mathbf{w}_{2}\right)\right|,\left|\tau_{m k}\left(\mathbf{w}_{3}\right)\right|,\left|\tau_{m k}\left(\mathbf{w}_{4}\right)\right|\right\}$ Else

$$
\begin{gathered}
\mathscr{F}\left(\mathbf{w}_{1}, \mathbf{w}_{2}, \mathbf{w}_{3}, \mathbf{w}_{4}\right)=0 \\
E_{\mathscr{L}}\left(\rho_{j m} \rho_{m k} \rho_{j n} \rho_{n k}\right)=\frac{1}{N^{4}} \\
\times \sum_{\mathbf{w}_{1}=1 \mathbf{w}_{2}=\sum_{3}=1 \mathbf{w}_{4}=1}^{L^{2}} \sum_{j m}^{L^{2}} \sum^{L^{2}} h_{1}\left(\mathbf{w}_{1}\right) h_{m k}\left(\mathbf{w}_{2}\right) h_{j n}\left(\mathbf{w}_{3}\right) h_{n k}\left(\mathbf{w}_{4}\right) \\
\times \mathscr{F}\left(\mathbf{w}_{1}, \mathbf{w}_{2}, \mathbf{w}_{3}, \mathbf{w}_{4}\right), \\
\tau_{j m}\left(\mathbf{w}_{1}\right)+\tau_{m k}\left(\mathbf{w}_{2}\right)=\tau_{j n}\left(\mathbf{w}_{3}\right)+\tau_{n k}\left(\mathbf{w}_{4}\right), \tau_{j m}\left(\mathbf{w}_{1}\right) \neq 0, \\
\tau_{m k}\left(\mathbf{w}_{2}\right) \neq 0, \tau_{j n}\left(\mathbf{w}_{3}\right) \neq 0, \tau_{n k}\left(\mathbf{w}_{4}\right) \neq 0, \\
\mathscr{\mathscr { F }}\left(\mathbf{w}_{1}, \mathbf{w}_{2}, \mathbf{w}_{3}, \mathbf{w}_{4}\right)=N \\
\text { Else if } \tau_{j m}\left(\mathbf{w}_{1}\right)+\tau_{m k}\left(\mathbf{w}_{2}\right)=\tau_{j n}\left(\mathbf{w}_{3}\right)+\tau_{n k}\left(\mathbf{w}_{4}\right), \\
\mathscr{F}\left(\mathbf{w}_{1}, \mathbf{w}_{2}, \mathbf{w}_{3}, \mathbf{w}_{4}\right)=N \\
-\max \left\{\left|\tau_{j m}\left(\mathbf{w}_{1}\right)\right|,\left|\tau_{m k}\left(\mathbf{w}_{2}\right)\right|,\left|\tau_{j n}\left(\mathbf{w}_{3}\right)\right|,\left|\tau_{n k}\left(\mathbf{w}_{4}\right)\right|\right\}
\end{gathered}
$$

Else

$$
\begin{gathered}
\mathscr{F}\left(\mathbf{w}_{1}, \mathbf{w}_{2}, \mathbf{w}_{3}, \mathbf{w}_{4}\right)=0 . \\
E_{\mathscr{L}}\left(\rho_{j m} \rho_{m k} \rho_{j k} \rho_{j j}\right)=\frac{1}{N^{4}}
\end{gathered}
$$

$\left.\times \sum_{\mathbf{w}_{1}=1 \mathbf{w}_{2}=1 \mathbf{w}_{3}=1 \mathbf{w}_{4}=1}^{L^{2}} \sum_{j m}^{L^{2}} \sum^{2}{ }_{\mathbf{w}_{1}}\right) h_{m k}\left(\mathbf{w}_{2}\right) h_{j k}\left(\mathbf{w}_{3}\right) h_{j j}\left(\mathbf{w}_{4}\right)$ 


$$
\begin{gathered}
\times \mathscr{F}\left(\mathbf{w}_{1}, \mathbf{w}_{2}, \mathbf{w}_{3}, \mathbf{w}_{4}\right), \\
\text { if } \tau_{j m}\left(\mathbf{w}_{1}\right)+\tau_{m k}\left(\mathbf{w}_{2}\right)=\tau_{j k}\left(\mathbf{w}_{3}\right), \tau_{j j}\left(\mathbf{w}_{4}\right)=0, \\
\mathscr{F}\left(\mathbf{w}_{1}, \mathbf{w}_{2}, \mathbf{w}_{3}, \mathbf{w}_{4}\right)=N \\
\times\left\{N-\max \left\{\left|\tau_{j m}\left(\mathbf{w}_{1}\right)\right|,\left|\tau_{m k}\left(\mathbf{w}_{2}\right)\right|,\left|\tau_{j k}\left(\mathbf{w}_{3}\right)\right|\right\}\right\} . \\
\text { Else if }\left|\tau_{j m}\left(\mathbf{w}_{1}\right)+\tau_{m k}\left(\mathbf{w}_{2}\right)-\tau_{j k}\left(\mathbf{w}_{3}\right)\right|=\left|\tau_{j j}\left(\mathbf{w}_{4}\right)\right|, \\
\tau_{j m}\left(\mathbf{w}_{1}\right) \neq 0, \tau_{m k}\left(\mathbf{w}_{2}\right) \neq 0, \tau_{j k}\left(\mathbf{w}_{3}\right) \neq 0, \\
\mathscr{F}\left(\mathbf{w}_{1}, \mathbf{w}_{2}, \mathbf{w}_{3}, \mathbf{w}_{4}\right)=N \\
-\max \left\{\left|\tau_{j m}\left(\mathbf{w}_{1}\right)\right|,\left|\tau_{m k}\left(\mathbf{w}_{2}\right)\right|,\left|\tau_{j k}\left(\mathbf{w}_{3}\right)\right|,\left|\tau_{j j}\left(\mathbf{w}_{4}\right)\right|,\right. \\
\left.\left|\tau_{j m}\left(\mathbf{w}_{1}\right)+\tau_{m k}\left(\mathbf{w}_{2}\right)\right|,\left|\tau_{j m}\left(\mathbf{w}_{1}\right) \pm \tau_{j j}\left(\mathbf{w}_{4}\right)\right|\right\} . \\
\text { Else if }\left|\tau_{j m}\left(\mathbf{w}_{1}\right)+\tau_{m k}\left(\mathbf{w}_{2}\right)-\tau_{j k}\left(\mathbf{w}_{3}\right)\right|=\left|\tau_{j j}\left(\mathbf{w}_{4}\right)\right|, \\
\mathscr{F}\left(\mathbf{w}_{1}, \mathbf{w}_{2}, \mathbf{w}_{3}, \mathbf{w}_{4}\right)=N \\
-\max \left\{\left|\tau_{j m}\left(\mathbf{w}_{1}\right)\right|,\left|\tau_{m k}\left(\mathbf{w}_{2}\right)\right|,\left|\tau_{j k}\left(\mathbf{w}_{3}\right)\right|,\left|\tau_{j j}\left(\mathbf{w}_{4}\right)\right|\right\} .
\end{gathered}
$$

Else

$$
\mathscr{F}\left(\mathbf{w}_{1}, \mathbf{w}_{2}, \mathbf{w}_{3}, \mathbf{w}_{4}\right)=0 .
$$

The first term is (108). The second term is (109).The third term is (110). The fourth term is (111). The fifth term is (112). The sixth term is (113).

\section{References:}

[1] S. Verdu, Minimum probability of error for asynchronous Gaussian multiple-access channels. IEEE Transactions on Information Theory.1986, Vol. IT-32, No. 1, pp. 85-96.

[2] S. Moshavi, Multiuser detection for DS-CDMA communications. IEEE Communications Magazine.1996, Vol. 34, No. 10, pp. 124-136.

[3] A. Duel-Hallen, J. Holtzman, Z. Zvonar, Multiuser detection for CDMA systems.IEEE Personal Communications. 1995, Vol. 2, No. 2, pp.4658.

[4] S. Verdu, Multiuser Detection. Cambridge, U.K. Cambridge University Press, 1998, 474 pages.

[5] Z Xie, R.T. Short, C.K. Ruthforth, A family of suboptimum detector for coherent multiuser communications. IEEE Journal of Selection Areas on Communications. 1990, Vol. 8, No. 4, pp.683 $-690$.

[6] R.M. Burhrer, N.S. Correal-Mendoza, B.D. Woerner, A simulation comparison of multiuser receivers for cellular CDMA. IEEE Transactions on Vehicle Technology. 2000, Vol. 59, No. 4, pp. 1065-1085.

[7] W. WU, W.S. Su, Yu-Tao, A two-stage MMSE partial PIC receiver for multiuser detection. In Proceedings 2001 IEEE Third Workshop on Signal Processing Advances in Wireless Communications (SPFWC'01). 2001, March 20-23, Taipei, Taiwan.

[8] R. Lupas, S. Verdu, Linear multiuser detectors for asynchronous code-division multiple-access channels. IEEE Transactions on Information Theory.1989, Vol. IT-35, No. 1, pp. 123-136.

[9] R. Lupas, S. Verdu, Near-far resistance of multiuser detectors in asynchronous channels. IEEE Transactions on Communications.1990, Vol. 35, No. 4, pp. 496-508.

[10] A.J. Viterbi, Very low rate convolutional codes for maximum theoretical performance of spread -spectrum multiple-access channels. IEEE Journal of Selection Areas on Communications. 1990 Vol. 8, No. 4, pp. 641-649.

[11] S.-C. Hong, J.Choi, Y.-H. Jung, Y.H. Lee, Constrained MMSE receivers for CDMA systems in frequency-selective fading channels. IEEE Transactions on Wireless Communications. 2004, Vol. 3, No. 5, pp. 1393-1398.

[12] J.M. Holtzman, Successive interference cancellation for direct sequence code division multiple access. IEEE Transactions on Communications. 1994, Vol. 19, No. 10, pp. 793-799.

[13] P. Patel, J.M. Holtzman, Analysis of simple successive interference cancellation scheme in DS-CDMA system. IEEE Journal of Selection Areas on Communications. 1994, Vol. 12, No.5 pp. 796-807.

[14] M. Ghotbi, M.R. Soleymani, Multiuser detection of DS-CDMA signals using parallel interference cancellation in satellite communications. IEEE Journal of Selection Areas on Communications.2004, Vol. 22, No. 3, pp. 584-593.

[15] M.K. Varanasi, B. Aazhang, Multistage detection in asynchronous code-division multiple-access communications. IEEE Journal of Selection Areas on Communications. 1994, Vol. 12, No.5 pp. 796-807.

[16] R.M. Buehrer, B.D. Woerner, Analysis of adaptive multistage interference cancellation for CDMA using an improved Gaussian approxiMation. IEEE Journal Transactions on Communications.1996,Vol.44, No.10, pp.1308-1320.

[17] R. Kohno, H. Imai, M. Hatori, S. Pasupathy, An adaptive canceller of co-channel interference for spread-spectrum multiple-access communication networks in a power line. IEEE Journal of Selection Areas on Communications. 1990, Vol. 8, No. 5, pp. 691-699.

[18] Y.C. Yoon, R. Kohno, H. Imai, A spread-spectrum multi-access system with co-channel interference cancellation. IEEE Journal of Selection Areas on Communications. 1993, Vol. 11, No. 7, pp. 1067-1075.

[19] Yu-Nao Hsien, Wen-Rong Wu, Performance analysis of an adaptive two-stage PIC CDMA receiver in AWGN channels. Signal Process- 
ing. 2008, Vol.88, No. 6, pp. 1413-1427.

[20] D. Divsalar, M.K. Simon, D. Raphaeli, Improved parallel interference cancellation for CDMA IEEE Transactions on Communications. 1998, Vol. 46, No. 2, pp. 258-268.

[21] N.S. Correal, R.M. Buehrer, B.D. Worner, A DSP-based DS-CDMA multiuser receiver employing partial parallel interference cancellation. IEEE Journal of Selection Areas on Communications.1999, Vol. 17, No. 3, pp. 613-630.

[22] J.-H. Wen, C.-W.Chang, H.-L.Hung, Multiuser detection using TGA-based partial parallel interference cancellation for CDMA systems. International Journal of Innovative Computing, Information and Control. 2009, Vol. 5, No. 9, pp. 2745-2767.

[23] M.J. Juntti, M. Latvaaho, Multiuser receivers for CDMA systems in Rayleigh fading channels. IEEE Transactions on Vehicle Technology. 2000, Vol. 49, No. 3, pp. 885-899.

[24] H. Chaonech, R. Bouallegue, Multiuser detection in asynchronous multibeam communications. International Journal of Wireless \& Mobile Networks. 2012, Vol. 4, No. 1, pp. 21-34.

[25] Y. Li, M. Chtn, S. Cheng, Determination of cancellation factors for soft-decision partial PIC detector in DS-CDMA systems. Electronics Letters. 2000, Vol. 36, No. 3, pp. 154-162.

[26] V. Tuzlukov, Signal processing by generalized receiver in DS-CDMA wireless communication systems with optimal combining and partial cancellation. EURASIP Journal on Advances in Signal Processing, published on-line on April 11, 2011, Vol. 2011, Article ID 913189, 15 pages, 2011, DOI:10.1155/2011/913189; 2011.

[27] H. Chaonech, R. Bonallegue, Multiuser detection and channel estimation for multibeam satellite communications. International Journal of Computer Networks \& Communications. 2012, Vol. 4, No. 1, pp. 163-174.

[28] G. Xue, J. Weng, T. Le-Ngoc, S. Tahar, Adaptive multistage parallel interference cancellation for CDMA. IEEE Journal of Selection Areas on Communications. 1999, Vol. 17, No. 8, pp. 1815-1827.

[29] R.M. Buehrer, S.P. Nicoloso, S.Gollamudi, Linear versus nonlinear interference cancellation Journal on Communications Networks. 1999 , Vol. 1, No. 2, pp. 118-133.

[30] J. Ravindrababu, E.V. Krishna Rao, Interference reduction in fading environment using multistage multiuser detection technique. WSEAS Transactions on Signal Processing. 2019, Vol. 15, Art. \#6, pp. 39-46.

[31] J. Ravindrababu, E.V. Krishna Rao, Y. Raja
Rao, Interference cancellation and complexity reduction in multistage multiuser detection. WSEAS Transactions on Communications. 2014, Vol. 13, Art. \#7, pp. 62-70.

[32] J. Wang, S. Zhang, W. Chen, D. Rjng, Z. Yu, Convex optimization-based multiuser detection in underwater acoustic sensor networks. International Journal of Distributed Sensor Networks. 2018, Vol. 14, Issue 2. DOI:10.1177/1550147718757665.

[33] D. Gou, L.K. Rasmussen, S. Sun, T.J. Lim, A matrix-algebraic approach to linear parallel interference cancellation in CDMA. IEEE Transactions on Communications. 2000, Vol. 48, No. 1, pp. 152-161.

[34] Y. Ren, C. Zhu, S. Xiao, Deformable faster R$\mathrm{CNN}$ with aggregating multilayer features for partially occluded object detection in optical remote sensing images. Remote Sensing. 2018, Vol. 10, Issue 9. DOI:10.3390/rs10091470.

[35] N.B. Mandayam, S. Verdu, Analysis of an approximate decorrelating detecto. Wireless Personal Communications. 1998, Vol. 6, No. 1-2, pp. 97-111.

[36] R.B. Buehrer, S.P. Nicoloso, Comments on partial parallel interference cancellation for CDMA IEEE Transactions on Communications. 1999, Vol. 47, No. 5, pp. 658-661.

[37] P.G. Renucci, B.D. Woerner, Optimization of soft interference cancellation for DS-CDMA. Electronic Letters. 1998, Vol.34, No. 8, pp. 731 $-733$.

[38] V. Tuzlukov, A new approach to signal detection theory. Digital Signal Processing. 1998, Vol. 8, No. 3, pp. 166-184.

[39] V. Tuzlukov, Signal Detection Theory, New York: Springer-Verlag, 2001, 725 pages.

[40] V. Tuzlukov, Signal Processing Noise, Boca Raton, London, New York, Washington, D.C.: CRC Press \& Francis Group. USA, 2002, 663 pages.

[41] V. Tuzlukov, Signal and Image Processing in Navigational Systems. Boca Raton, London, New York, Washington, D.C.: CRC Press \& Francis Group. USA, 2005, 635 pages.

[42] M. Maximov, Joint correlation of fluctuative noise at outputs of frequency filters. Radio Engineering. 1956, No. 9, pp. 28-38.

[43] Y. Chernyak, Joint correlation of noise voltage at the outputs of amplifiers with no overlapping responses. Radio Physics and Electronics. 1960, No. 4, pp. 551-561.

[44] V. Tuzlukov, DS-CDMA downlink systems with fading channel employing the generalized detector. Digital Signal Processing, 2011. Vol. 
21, No. 6, pp. 725-733.

[45] V. Tuzlukov, Signal processing by generalized detector in DS-CDMA wireless communication systems with frequency-selective channels. $\mathrm{C}$ rcuits, Systems, and Signal Processing. 2011, Vol. 30, No. 6, pp.1197-1230; published online on February 2, 2011, doi:10.1007/s00034 -011-9273-1.

[46] M. Shbat and V. Tuzlukov, Primary signal detection algorithms for spectrum sensing at low SNR over fading channels in cognitive radio. Digital Signal Processing (2019). https://doi. org/10.1016/j.dsp. 2019.07.16. Digital Signal Processing. 2019. Vol.93. No.5, pp.187- 207.

[47] Shbat, M., Tuzlukov, V.P, Evaluation of detection performance under employment of the generalized detector in radar sensor systems. Radioengineering, 2014, Vol. 23, No. 1, pp. 50-65.

[48] M. Shbat and V. Tuzlukov, Definition of adaptive detection threshold under employment of the generalized detector in radar sensor systems. IET Signal Processing, 2014, Vol. 8, Issue 6, pp. 622-632.

[49] M. Shbat and V. Tuzlukov, SNR wall effect alleviation by generalized detector employment in cognitive radio networks. Sensors, 2015, 15 (7), pp.16105-16135;doi:10.3390/s 150716105.

[50] V. Tuzlukov, Signal processing by generalized receiver in wireless communications systems over fading channels. Chapter 2 in Advances in Signal Processing. IFSA Publishing Corp. Barcelona, Spain. 2021. pp. 55-111. https://doi.org/10.1038/s41598-021-84139-3

[51] M. Shbat, V. Tuzlukov, Generalized detector as a spectrum sensor in cognitive radio networks. Radioengineering , 2015, Vol. 24, No. 2, pp. 558-571.

[52] D.R. Brown, M. Matani, V.V. Veeravalli, Y.V. Poor, C.R. Johnson, On the performance of linear parallel interference cancellation. IEEE Transactions on Information Theory. 2001, Vol.47, No. 7, pp. 1957-1970.

[53] D. Gou, S. Verdu, L.K. Rasmussen, Asymptotic normality of linear multiuser receiver outputs. IEEE Transactions on Information Theory. 2002, Vol.48, No. 12, pp. 3080-3095.

[54] H.V. Poor, S. Verdu, Probability of error in MMSE multiuser detection. IEEE Transactions on Information Theory. 1997, Vol.43, No. 5, pp. 858-871.

[55] S.R. Kim, I. Choi, S. Rang, J.G. Lee, Adaptive weighted parallel interference cancellation for CDMA systems. Electronics Letters. 1998, Vol. 34, No. 22, pp. 2085-2086.
[56] V. Tuzlukov, Optimal combining, partial cancellation and channel estimation and correlation in DS-CDMA systems employing the generalized detector. WSEAS Transactions on Communications. 2009, Vol. 8, Issue 7, pp.718-733.

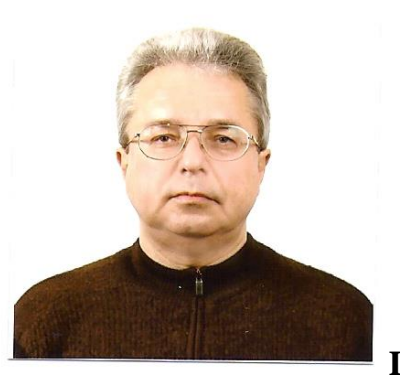

Dr. Vyacheslav Tuzlukov received the MSc and $\mathrm{PhD}$ degrees in radio physics from the Belarusian State University, Minsk, Belarus in 1976 and 1990, respectively, and DSc degree in radio physics from the Kotelnikov Institute of Radioengineering and Electronics of Russian Academy of Sciences in 1995. Starting from 1995 and till 1998 Dr. Tuzlukov was a Visiting Professor at the University of San-Diego, San-Diego, California, USA. In 1998 Dr. Tuzlukov relocated to Adelaide, South Australia, where he served as a Visiting Professor at the University of Adelaide till 2000. From 2000 to 2002 he was a Visiting Professor at the University of Aizu, Aizu-Wakamatsu City, Fukushima, Japan and from 2003 to 2007 served as an Invited Professor at the Ajou University, Suwon, South Korea, within the Department of Electrical and Computer Engineering. Starting from March 2008 to February 2009 he joined as a Full Professor at the Yeungnam University, Gyeonsang, South Korea within the School of Electronic Engineering, Communication Engineering, and Computer Science. Starting from March 1, 2009 Dr. Tuzlukov served as Full Professor and Director of Signal Processing Lab at the Department of Communication and Information Technologies, School of Electronics Engineering, College of IT Engineering, Kyungpook National University, Daegu, South Korea. Currently, Dr. Tuzlukov is the Head of Department of Technical Exploitation of Aviation and Radio Engineering Equipment, Belarusian State Academy of Aviation, Minsk, Belarus. His research emphasis is on signal processing in radar, wireless communications, wireless sensor networks, remote sensing, sonar, satellite communications, mobile communications, and other signal processing systems. He is the author over 280 journal and conference papers, eighteen books in signal processing published by Springer-Verlag and CRC Press. Some of them are Signal Detection Theory (2001), Signal Processing Noise (2002), Signal and Image Processing in Navigational Systems (2005), Signal Processing in Radar Systems (2012), Editor of the book Communication Systems: New Research (2013), Nova Science Publishers, Inc, USA, and has also contributed Chapters "Underwater Acoustical Signal Processing" and "Satellite Communications Systems: Applications" to Electrical Engineering Handbook: $3^{\text {rd }}$ Edition, 2005, CRC Press; "Generalized 
Approach to Signal Processing in Wireless Communications: The Main Aspects and Some Examples" to Wireless Communications and Networks: Recent Advances, InTech, 2012; "Radar Sensor Detectors for Vehicle Safety Systems" to Electrical and Hybrid Vehicles: Advanced Systems, Automotive Technologies, and Environmental and Social Implications, Nova Science Publishers, Inc., USA, 2014; "Wireless Communications: Generalized Approach to Signal Processing" and "Radio Resource Management and Femtocell Employment in LTE Networks", to Communication Systems: New Research, Nova Science Publishers, Inc., USA, 2013; "Radar Sensor Detectors for Vehicle Safety Systems" to Autonomous Vehicles: Intelligent Transport Systems and Automotive Technologies, Publishing House, University of Pitesti, Romania, 2013; "Radar Sensor Detectors for Vehicle Safety Systems," to Autonomous Vehicles: Intelligent Transport Systems and Smart Technologies, Nova Science Publishers, Inc., New York, USA, 2014; "Signal Processing by Generalized Receiver in DS-CDMA Wireless Communication Systems," to Contemporary Issues in Wireless Communications. INTECH, CROATIA, 2014; "Detection of Spatially Distributed Signals by Generalized Receiver Using Radar Sensor Array in Wireless Communications," to Advances in Communications and Media Research. NOVA Science Publishers, Inc., New York, USA, 2015; "Signal Processing by Generalized Receiver in Wireless Communications Systems over Fading Channels" to Advances in Signal Processing. IFSA Publishing Corp. Barcelona, Spain. 2021.

He participates as the General Chair, Keynote Speaker, Plenary Lecturer, Chair of Sessions, Tutorial Instructor and organizes Special Sections at the major International Conferences and Symposia on signal processing.

Dr. Tuzlukov was highly recommended by U.S. experts of Defence Research and Engineering (DDR\& E) of the United States Department of Defence as a recognized ex- pert in the field of humanitarian demining and minefield sensing technologies and had been awarded by Special Prize of the United States Department of Defence in 1999 Dr. Tuzlukov is distinguished as one of the leading achievers from around the world by Marquis Who's Who and his name and biography have been included in the Who's Who in the World, 2006-2013; Who's Who in World, 25 ${ }^{\text {th }}$ Silver Anniversary Edition, 2008, Marquis Publisher, NJ, USA; Who's Who in Science and Engineering, 20062012 and Who's Who in Science and Engineering, 10th Anniversary Edition, 2008-2009, Marquis Publisher, NJ, USA; 2009-2010 Princeton Premier Business Leaders and Professionals Honours Edition, Princeton Premier Publisher, NY, USA; 2009 Strathmore's Who's Who Edition, Strathmore's Who's Who Publisher, NY, USA; 2009 Presidental Who's Who Edition, Presidental Who's Who Publisher, NY, USA; Who's Who among Executives and Professionals, 2010 Edition, Marquis Publisher, NJ, USA; Who's Who in Asia 2012, $2^{\text {nd }}$ Edition, Marquis Publisher, NJ, USA; Top 100 Executives of 2013 Magazine, Super Network Publisher, New York, USA, 2013; 2013/2014 Edition of the Global Professional Network, Business Network Publisher, New York, USA, 2013; 2013/2014 Edition of the Who's Who Network Online, Business Network Publisher, New York, USA, 2014; Online Professional Gateway, 2014 Edition, Business Network Publisher, New York, USA, 2014; 2014 Worldwide Who's Who", Marquis Publisher, NJ, USA; 2015 Strathmore Professional Biographies, Strathmore's Who's Who Publisher, NY, USA; Who's Who in World, 2015, Marquis Publisher, NJ, USA; 2015-2016 Membership in Exclusive Top 100 network of professionals in the world, NY, USA, 2015; 2015 Who's Who of Executives and Professionals Honors Edition, Marquis Publisher, NJ, USA; Worldwide Who's Who - Top 100 Business Networking, San Diego, CA, USA, 2015.

Phone: +375173453283

Email: slava.tuzlukov@mail.ru

\section{Creative Commons Attribution License 4.0} (Attribution 4.0 International, CC BY 4.0)

This article is published under the terms of the Creative Commons Attribution License 4.0 https://creativecommons.org/licenses/by/4.0/deed.en_US 